\author{
UCRL-10431 \\ MASTEF \\ University of California \\ Ernest 0 . Lawrence \\ Radiation Laboratory
}

\title{
LINEAR STOCHASTIC MOTIONS OF PHYSICAL SYSTEMS
}

Berkeley, California 


\section{DISCLAIMER}

This report was prepared as an account of work sponsored by an agency of the United States Government. Neither the United States Government nor any agency Thereof, nor any of their employees, makes any warranty, express or implied, or assumes any legal liability or responsibility for the accuracy, completeness, or usefulness of any information, apparatus, product, or process disclosed, or represents that its use would not infringe privately owned rights. Reference herein to any specific commercial product, process, or service by trade name, trademark, manufacturer, or otherwise does not necessarily constitute or imply its endorsement, recommendation, or favoring by the United States Government or any agency thereof. The views and opinions of authors expressed herein do not necessarily state or reflect those of the United States Government or any agency thereof. 


\section{DISCLAIMER}

Portions of this document may be illegible in electronic image products. Images are produced from the best available original document. 
UCRL- 1043.1

UC-34 Physics

TID-4500 (17th Ed.)

\author{
UNIVERSITY OF CALIFORNIA \\ Lawrence Radiation Laboratory \\ Berkeley, California \\ Contract No. W-7405-eng -48
}
LINEAR STOCHASTIC MOTIONS OF PHYSICAL SYSTEMS
Ching-Hung Woo
(Ph.D. Thesis).
August 1962

Reproduced by the Technical Information Division directly from Author's copy. 
Printed. in USA. Price \$1.50. Available from the office of Technical Services U. S. Department of Commerce Washington 25, D.C. 


\section{CONDENTS}

I. INTRODDUCTION

A. Stochastic Motions and Diffusion on a Group Manifold

B. Some Physical Kodels for Stochastic Notions

II. THE SET OF DENSTTY IMTRICES

III. LINWA STOCHASTIC NOTIONS

A. Types of Motions

B. Conditions for the sets $\mathcal{\&}, \mathcal{Q}_{I}, \mathcal{Q}_{\mathcal{I}}$

C. The Disordering Nature of Kotions of Type (B)

D. Stable Ensemules

IV. PROPERTIES OF STOCHASTIC IOTIONS WIIC! PRESERVE I

A. Unitary and Antiunitary Transformations on $\underset{\sim}{V}$

B. Norms of Vectors in $\stackrel{V}{\sim}$ under Nappings in $\mathscr{P}_{I}$

c. Properties of $\mathbb{P}_{I}$ as a Conver Set

V. THE DIFTUSION EQUATIOLS

A. Derivation of the Diffusion Equations

B. Normalization and Integral Identities for $\mathrm{P}(g ; t)$

C. Diffusion Equation and lotions of Type (B)

VI. WURTHER STUDIES RELATED TO THE DIFTUSON ECUATIONS

A. Definition of Entropies

B. Possible Reduction of the Diffusion Equation for $P(g ; t)$

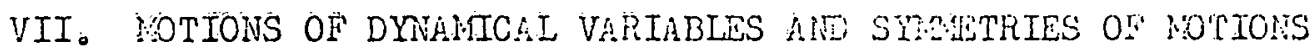

A. Tine Development or Dymamical Vuriables

B. Symmetries Possessed by lotions 


\section{LINEAR STOCHASIIC MOTIONS OF PHYSICAL SYSTEMS \\ Ching-Hung Woo.

\author{
Lawrence Radiation Laboratory \\ University of California \\ Berkeley, California
}

August, 1962

ABSTRACT

A study is made of some aspects of Linear Stochastic Motions in quantum mechanics and classical mechanics. Such motions are characterized by the feature that a linear transformation, describes the time development of the density matrix representing the ensemble in quantum mechanics, or of the density distribution function on phase space representing the ensemble in classical mechanics. The discussion is mostly limited to Disordering Stochastic Motions, by which are understood stochastic motions, which preserve the uniform ensemble; such motions are shown never to lead to an increase in information about the ensemble. As a special case disordering stochastic motions which can be interpreted as corresponding to a diffusion process on a finite dimensional Lie group are considered; for such processes the close analogy between the description in quantum mechanics and the description in classical mechanics is stressed. The equivalence. between various equations of motion which have been considered in the Iiterature is discussed; this discussion is mostly limited to quantum mechanical systems which can be described on finite dimensional Hilbert spaces. For disordering stochastic notions of such systems the set of all time development transformations form a closed, bounded, convex set. It is shown that the set of extreme points of this set includes transformations which are neither unitary nor antiunitary. 


\section{INRKDUCTION}

\section{A. Stochastic Motions and. Diffusion on a Group Manifold}

In this section we wish to emphasize a certain unifying vievpoint concerning the equation of riotion of physical systems.

Let us consier first the motion of a quantum rnechanical system within the franevork of Haniltonian aechanics. The systen at time $t$ is related so the system at tirie zero by a unitary transformation $U^{\prime}(t)$. If the untery transformations generate a finite dimensional lie group, then $t$ t.e transformation $U(t)$ can be labeled by the corresponding group elems..s, i.e., by a point on the group manifold. The time developinent of 1 system is then describec by a continuous curve on the group mani-

fois. ${ }^{1}$ An exactly analogous situation prevails in the case os a classical syetem. Here one may consider the time developnent of points in phaseepace. The systein at time $t$ is related to the original system by a contact tiensformation. Again if the contact transformations generate a rinite dimensional lie group, the state of the systen can be labeled by a point on the group manirold. The cssence of the notions is thus in both cases the curve on the group manifold. A detailed exposition of this well-established principle, as nil se sone of its jouctical applications, has been presented in the renencuce quoted above. I

We now consider the extension of this vicupoint to the case ot an enswble of systems undergoing random transfowntions. In this case, instead of describing a continuous curve on the group manifold, the system will have various probabilities of going in different directions at each point of its pich. In each direction further branching wil:

I. E. H. Wichmann, J. Wath. Fnys, 2, 100.6,5\%0 (1961). 
occur. The time developient corresponds, therefore, to a diffusion process on the group manivid. The advantages of such a viewpoint, besides serving as a connectirg link between quartum mechanics and classical mechanics, consist in separating out from the problem the part which depends only on the structure of tre group, and not on its specific reallation on the hilbert space $\underline{1}$ of the stato vection of the systern. Group theoreicil methods may also help to simplify the integration problem ir sowe cases.

The jeneral description of the evolution of an ensemble of systems, in accorcince with the above viewpoint, will then be as follows. The enseridis arter time $t$ is to be considered a mixture of ensembles obtained. frcis the original ensenble at $t=0$ by all possible transformations in thes time interval. We consider in particular the case where the group Eenerated by these transpormations is a finite dimensional lie group. $Y$, and denote a general element of $\mathscr{Y}$ : by $g$. The amount by which the verious transforned enewiles enter the mixtire vill be deternined by a probability distribution furction, wich we denote $P(\varepsilon ; t)$. It is of : interest the: to find the differential equation scisilied by $P(g ; t)$. when the rotion is sufficiently contrivous. The derivation of suct an equation, arci tine study of its relation to the differential equation satisfied by the density matrix $f(t)$ of the system, or the differertial equation of the phase-spece dersity. function of a classical systcm, will be the subject of section $V$.

B. Some Physical Rodels for Stocrastic rotions

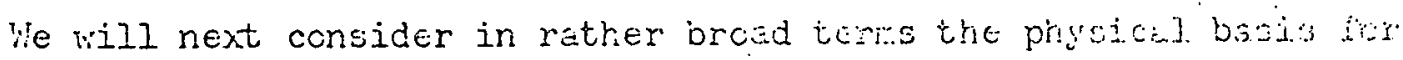


the ways whereby the information about a system may be lost; ard we will analyze two types of processes, which should have rather gerieral relevance.

(a) The system is subject to randori external forces of such a nature that the process becomes larkovian. 2 An exemple of such a process is provided by a gas of particles with magnetic moments in a container, over the volume of which the magnetic field fluctuates in space. Furthermore, it may fluctuate in a randon way in time. For such processes it is clear that the motion, in spin space, is a superposition of unitary trarsformations. One may say that the stochastic nature of the motion is a consequence of randomly fluctuating (classical) external forces.

(b). The second case arises as follows: Let us denote the elcment of an ensemble by $S$, and the ensenble itself by (S). Each element can urdergo the following sequence of events: at a certain rardomly determined tine it gets irto a close interaction, a "collision", with arother quaroum mechanical system $\mathrm{R}_{s}$ which is an elencht in ariother ensemble ( $\mathrm{P}$ ). This latter may be considured the reservoir if it is a conperatively "Iargeli systen; but need not be so considered in the general case. The combined syeten s-k then undergoes a unitary trineformation for sone fixed small tine, $\dot{i}_{0} e_{.}$, sriall compared to the average time spent for a measurement; after wich $S$ and $R$ cease to interact. In the case where $(R)$ is considered a reservoir, the nurnber of syctens ir. (i) is assumed to be so large that the state of the reservoir does nct El.: with tin:e because of the interaction with the systems in (s); for the stationery case one essures that (R) does not change with tinc cue

2. Hakcvien in the serse thet the system at tine t" is uniouely deter-

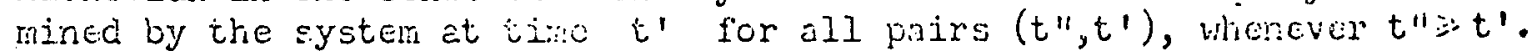


to any other cause either. Suppose now one tries to describe this situation: At the beginning of a collisicr: event both $S$ and $R$ are described by vectors $s_{k}$ and $r_{\mu}$ in the apropriate nilbert spaces, which need not be of the same dimensionglity. Lev $U$ be the unitary transformation which describes what happens curing the eveni; it acts on the tensor product of two Hilbert spaces, and may in some version be assumed to be a fixed transformtico. The rinal state vector for the corabined system is no longer a product of two vectors describing $S$ and $R$ separately, but is given iy

$$
\because_{n \lambda}=\sum_{n, \nu} U_{m \lambda, n \nu} s_{n} r_{\nu}
$$

It ve now form a reeuced unsemble ${ }^{3}$ nich cescribes the result of

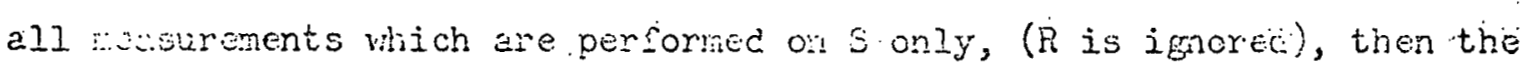
dereity natrix describing the systa $S$ after the tranefornation is

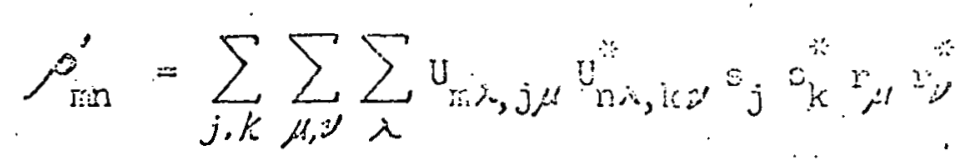

This describes an individual ovet. Coneider now the change in the enswble (s) curing a time which is so eicre that the sane cleneme $s$ in (s) rever undergoes more than one coliteion, weress the tine is long compered to the collision time. Iet the initial stete of (s) be given by the dencity matrix $\rho$, and the final stite by $\rho^{\prime \prime}$. Let the state

3. Fon dny density matrix $\rho_{\text {ma,n }}$ descriome che combined systen, is : is

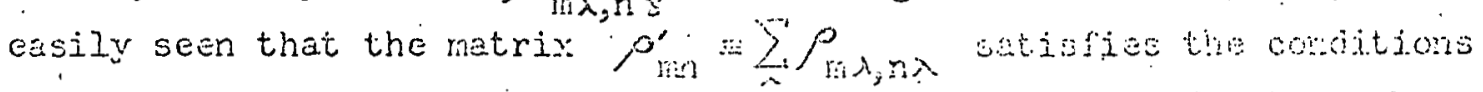
of a density matrix, and is cellec the reduces clechlo for the sub syetern (S). 
of $(R)$, which is taken to be constant for a reservoir, be given by the density matrix $\omega$. We then have, in accordance with the result for a single event,

$$
\rho_{m n}^{\prime \prime}=(1-c) \rho_{m n}^{\beta}+c \sum_{j, k} \sum_{\mu, \nu} \sum_{\lambda} U_{m, j \mu} v_{n \lambda, k \nu}^{*} \rho_{j k} c v_{\mu \nu}
$$

where the real constant $c$ satisfies $1>c>0$, and is proportional to the number of events: It is to be noticed that the resultine transfomation on an individual state vector describing an element $S$ of: (S) is linear: there is no complex conjugation.

Cre has a slightly more general situation if we also carry out a randonization of $\mathrm{U}$ in $\mathrm{Eq}$. ( $\mathrm{I}-3$ ). But apart from this the equation should have rather general validity in describing the physical basis. for the possible loss of information about (s): The total infornetion about the corbined system remins unchanged by a unitary transformation; after the transiormation, part of the information resides in the correlation between $(S)$ and $(R)$. This information is thrown away when the correlation is not observed. The enscmble (R) is also not observed either before or after the transformation. So if the information which one can in principle have about $(R)$ is increased, or remains unchanged, by the transformation, the information which one can have about (S) must decrease. On the other hand, if the infornation which one cen have about $(R)$ decreases through the transformation by such an ariount as to exceca the loss of information in correlation, then the inforration about (s) may yet increase.

We will show later that if one requires that the uniform enemble 
$\rho=I / \operatorname{Tr}(I)$ in (S) be preserved by the transformation, this puts such a strong requirenert on $(R)$ and $U$ that the information atiout $(S)$ is necessarily non-ncreasing.

At this point we pause to indicate the direction, and to discuss the motivation, of the analysis in the sections to follow.

We ficto scen that there is a natural correspondence between some stochestic motions and a diffusion process on a group manifold. We have also scen examples of two kinds of physical processes of a rather general netine thereby the information about a system may be lost. While the process described in (a) clearly falls within the scope of the diffusion description, the process of type (b) as exemplified by Eq. (I-3) does not have such a direct correspondence. Cur analyeis will therefore be twofold: Ca the one hand we will analyze the most general linear rnotions which transform any density matrix into another density matrix, and such that the information which one may have about the eneemble represented by the density matrix is decreased or at lesst undianged by the transformation (sections III and IV); on the otleer hand, in view of the physical simplicity of the diffusion description, and the fact that there are. many processes which naturally fall under this category, we will aiso Give a separate treatment of the diffusion equations (sections V and VI). The relations between the two framevoriks are disciseed in the subsections $I V-C$ and $V-C$. 


\section{THE SET OF DENSITY MATRICES}

Before we proceed we wish to review briefly some properties of the set of density matrices, and to estalblish some notations.

In order to avoid complicitions wich it is not the purpose of this work to discuss, we will linjt ourselves to finit. - dinensional Hilbert spaces. Some remaits about extensions to infinite-dinengionsl Hilbert. spaces will bie macio later.

- Vis will give a ljst of the notations wich will be used the most often: (a) V is the vactior space of all Min metrices. These matrices will be cennter by block canjtals, with the exception of rensity matrices, which vi].I bi denotiù by $\rho$ or $\omega$.

$\underline{V}_{h}$ is the real vector spare of all inemitean lixn matricos. (b) Hemplean idenpotentis in $\mathrm{V}$ are denoted ry $F$; thus $F^{+}=F, F F=\vec{F}$. Hemitean prinitive idemedents abo denoted by $\mathrm{E}$; thus $\mathrm{E}^{+}=\mathrm{E}$, $\operatorname{Tr} \Gamma=1, \mathrm{BE}=\mathrm{F}$.

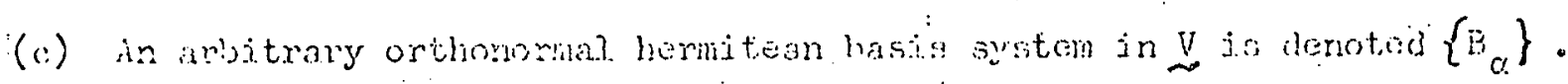

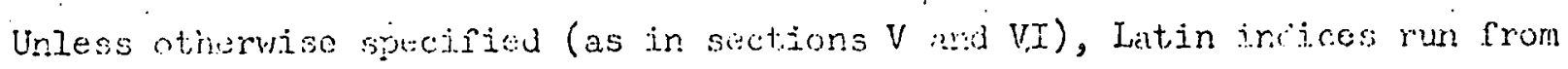

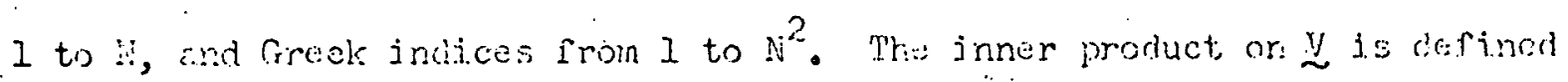
by the: trace operation; thus $B_{\alpha}^{+}=B_{\alpha}, \operatorname{Tr}\left(B_{\alpha} B_{\beta}\right)=\delta_{\alpha \beta}$, and

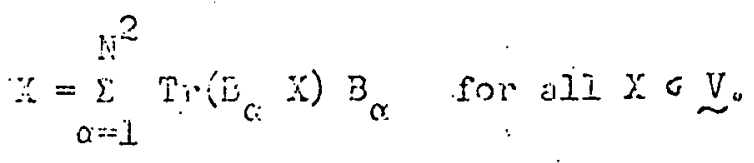

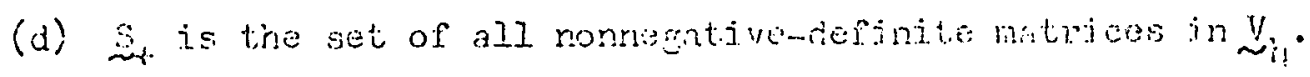

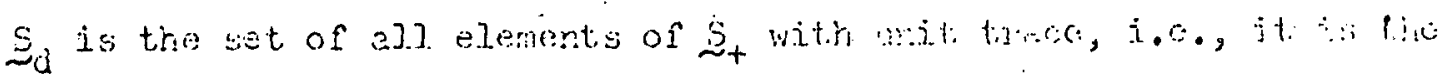
sot of all Mal density matrjecs.

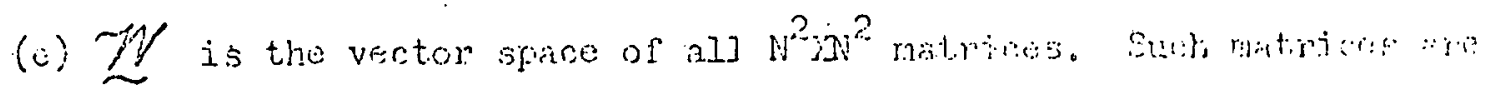
denoteci oy cepital sorjot letters.

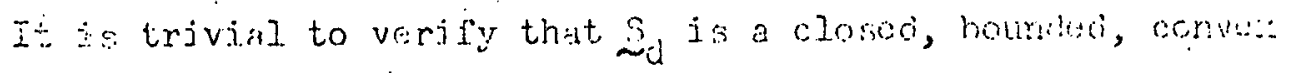

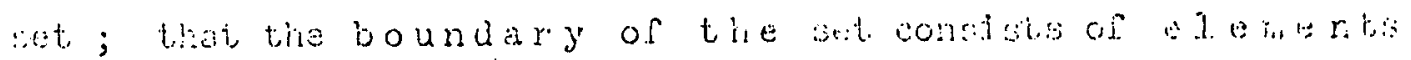


of ${\underset{\sim}{d}}_{\mathrm{d}}$ vith vanishing determinants, and the extrene points consist precisely of all primitive hermitean idempotents. 4,5

The set $\Phi_{d}$ can be partially ordered. Consider two sets of nonnegative numbers $\left\{x_{k}\right\},\left\{y_{l}\right\}$, with $\sum_{k=1}^{N} x_{k}=\sum_{k=1}^{N} y_{k}=1_{0}$ We denote

$$
\{x\} \leqslant\{y\}
$$

If the numbers can be so arranged as to satisfy the following:

$$
x_{1} \geqslant x_{2} \geqslant \ldots \geqslant x_{N} \geqslant 0 ; \quad y_{1} \geqslant y_{2} \geqslant \cdots \geqslant y_{N} \geqslant 0
$$

and,

$$
\sum_{k=1}^{p} x_{k} \leqslant \sum_{k=1}^{p} y_{k} \quad \text { for } p=1,2, \ldots, N
$$

It can be shom ${ }^{6}$ that $\{x\}\{y\}$ is completely equivalent to the existence of a real matrix $D$ such that $\underset{\sim}{*}=\dot{D} \underset{\sim}{\mathrm{y}}$, and

$$
D_{m k} \geqslant 0 ; \quad \quad \quad \sum_{k=1}^{N} D_{n k}=\sum_{m=1}^{N} D_{r k k}=1
$$

Such a matrix is called a doubly stochastic natrix.

4. For ierninologies and basic facts about convex sets, we refer the reader to the book by H. G. Egegeston, Convexity, (Cambrided University Press, Cambridge, 1958). An extrene point $x$ of a conw: set is one such that it is impossible to find wo points in the $a$ different from $x$ and such that $x$ lie; on the line serkent joining these two points.

5. See also ?. F. Jordan and E. C. G. Sudarshar, J. Hath. Mhys., 2,772 (I96I); and E. C. G. Sudarshan, P. H. Mathens, and J. Ratu, Hys. Kev., 121,920 (2.961).

6. G. H. Wardy, J. E. Littlewood, and G. Polya, Memmitins, (Coibidge University Press, Cambridge, 1959), 2nd ed., Chap. ?, 9.4\% 
We now introduce the following partial ordering. Let $\rho$ and $\rho^{\prime \prime}$ be in $\underline{s}_{d}$, and let $\left\{r_{k}^{\prime}\right\}$ and $\left\{r_{k}^{\prime \prime}\right\}$ be their eigenvalues, respectively. We denote

$$
\rho^{r} \leqslant \rho^{\prime \prime}
$$

if

$$
\left\{r^{\prime \prime}\right\} \leqslant\left\{r^{\prime \prime}\right\}
$$

If the possibility of $\left\{r_{k}^{\prime}\right\}=\left\{r_{k}^{\prime \prime}\right\}$ is excluded, we write.

$$
\rho^{\prime}<\rho^{\prime \prime}
$$

For each $\rho$ in $S_{d}$, one can define the entropy corresponding to the ensemble which it represents by

$$
s=-\operatorname{Tr}(\rho \ln \rho)
$$

As pointed out by Von Neumarn ${ }^{7}$, if $f(x)$ is any other continuous strictiy convex sunction ${ }^{8}$, then - Tr $\rho(\rho)$ has equally acceptable properties as a
7. J. von Newrann, Watheratical Foundatjons of Qunturn Wochonics, (jrin- cetcon Unlversity Press, Princeton, 1955), translated by R. T. Ecyer, Ciap. V, p. 390.

8. We rill denote by $F$ the set of all continuolis strictly conver furctions $r(x)$, defined for $x>0$; $1 . e$., for ariy $f(x) \in F$, and any $\theta$ such that $0<\theta<1$, cine has

$$
f\left(\theta x_{1}+(1-\theta) x_{2}\right)<0 f\left(x_{1}\right)+(1-\theta) f\left(x_{2}\right)
$$

for all $x_{1}$ and $x_{2}$ such that $0<x_{1}<x_{2}$. 
measure of randomness or lack of information for non-chermodynainical irreversible considerations. Now a theoren ${ }^{9}$ in reference 6 states the following:

Given any two sets ô real non-reeative numbers $\left\{x_{k}\right\},\left\{y_{k}\right\}$, with $\sum_{k=1}^{N} x_{k}=\sum_{k=1}^{N} y_{k}=1$, in order that for every $f(x)$ in $F_{c}$

$$
\sum_{k=1}^{N} f\left(x_{k}\right) \leqslant \sum_{k=1}^{N} f\left(y_{k}\right)
$$

it is necessary and sufficient that $\{x\} \leqslant\{y\}$.

This important theoren shows that, if $\rho^{\prime} \leqslant \rho^{\prime \prime}$, then the ensemble represented by $\rho^{\prime}$ contains generally less information than that represented by $\rho^{\prime \prime}$.

From Eq. (II-9) one can obtain Von Neumann's mixing theorem ${ }^{10}$;

Let $\hat{I}(x) \in F_{c} ; \quad \rho^{\prime}$ and $\rho^{\prime \prime} \in S_{d} ;$ and let $\theta$ beresi and such that $0<0<1$; then

$$
0 \operatorname{Tr} r\left(\rho^{\prime}\right)+(I-\theta) \operatorname{Tr} r\left(\rho^{\prime \prime}\right) \geqslant \operatorname{Tr} r\left(\theta \rho^{\prime}+\left(1 .-\theta^{\prime}\right) \rho^{\prime \prime}\right)
$$

the equality holds if and only if $\rho^{\prime}=p^{\prime \prime}$

Ve will give a proof somewat differont fron the ore hintud in ron Neunarn's book. Consider the expansion of $\rho^{\prime}, p^{\prime \prime}$, and $p=0, \rho^{\prime}+(1-0) p^{\prime \prime}$

9. Reference 6, Cnap. 3, p. 89.

10. Reference 7, Cnap. V., p. 390. 
น

Into pidmltive hornitosin Idempotontiol

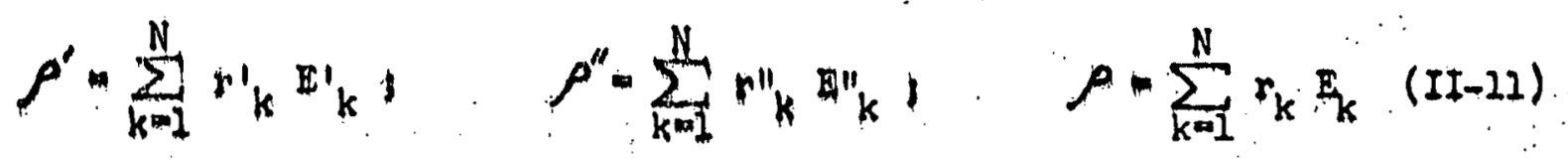

Hence

$$
r_{n}-\sum_{k=1}^{N}\left[\theta r_{k}^{\prime} \operatorname{Tr}\left(E_{k}^{\prime} \cdot E_{n}\right)+(1-\theta) r_{k}^{\prime \prime} \operatorname{Tr}\left(E_{k}^{\prime \prime} E_{n}\right)\right]
$$

Since

$$
\theta \operatorname{Tr}\left(E_{k} E_{n}\right)>0, \quad(1-\theta) \operatorname{Tr}\left(E_{k} \|_{n}\right) \geqslant 0
$$

and

$$
\sum_{k=1}^{N}\left[\theta \operatorname{Tr}\left(E_{k} E_{n}\right)+(\mathcal{L}-\theta) \operatorname{Tr}\left(E_{k} E_{n}\right)\right]: 1
$$

we Imrnedlately obtain, for $f(x)$ in $F_{c}$,

$$
\sum_{n=1}^{N} f\left(r_{n}\right) \leqslant \sum_{n, k=1}^{N}\left[\theta f\left(r^{\prime}{ }_{k}\right) \operatorname{Tr}\left(E^{\prime}{ }_{k} E_{n}\right)+(1-\theta) f\left(r^{\prime \prime}\right) \operatorname{Tr}\left(E_{k} E_{k}\right)\right]
$$

that is,

$$
\sum_{n=1}^{N} f\left(r_{n}\right) \leqslant \quad \sum_{k=1}^{N}\left[\theta f\left(r_{k}^{\prime}\right)+(1-\theta) f\left(r_{k}^{\prime \prime}\right)\right]
$$

which 18 the assertion (II-10) of the thearem. Since $f(x)$ is strictly convex the equality alen can obvloubly obtain only if ${ }^{\prime} \rho=\rho^{\prime \prime}$.

It follows frorn Eq. (II-10) that for any closed convex subset of sij' there axist a undgu $\rho_{e}$ such that for any other $\rho$ in the subset; 
and for all $f(x) \in F_{c}$

$$
\operatorname{Tr} f(\rho)>\operatorname{Tr} f\left(\rho_{0}\right)
$$

In the particular case where the sot is equal to $\Phi_{\mathrm{d}}$ itself $\rho_{0}=I / N$, the uniform enfemble. For the unf form ensemble evory state is equally. probable; it thus contains the least information. On the other extreme; one has the primitive herruitean idempotents $I$ which correspond to pure states, 'It can readily be show that for any $\rho \in S_{d}$, and $f(x) e f$;

$$
f(1)+(N-1) f(0)=\operatorname{Tr} f(E) \geqslant \operatorname{Tr} f(\rho)
$$

where the equality sigl holds if and only if $\rho$ is another primitive heraitean idempotent. 
15

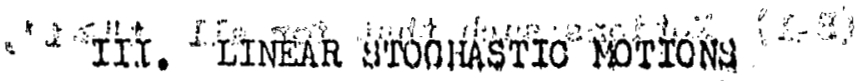

A. Typo: of lifitions : is :

Havilug reviewed tho properties of the set $S_{d}$, wo will now consider Inear mappings of the sot $S_{\mathrm{d}}$ into itself.

We denota by $\mathcal{P}$ the set of all matrices in $\mathscr{W}$. which map $s_{d}$ into $S_{d}$; theso mapplngs were named dynamical mappings in ref. 5. Tho set of all matrices in $\&$ which preserve the uniform ensemble will bo denoted by $\mathscr{U}_{I^{*}}$ It is to this latter set of mappings that we will devote most of our attentions, with only occasional roforence to \&.

Corresponding to these mappings are motions; i.e., one may consider oquations of the type: $\rho\left(t^{\prime \prime}\right)=\mathcal{R}\left(t^{\prime \prime}, t^{\prime}\right) \rho\left(t^{\prime}\right)$, with $R\left(t^{\prime \prime}, t^{\prime}\right) \in \mathcal{S}$ or $\mathscr{U}_{I}^{\prime}$ for all $t^{\prime \prime} \geqslant t^{\prime}$. We will study these together with motions of other varleties, which we will now enumarate. The reasons for the inclusion of particular types of riotions will become clearer through the later discussion.

$(A-1)$ Potions such thet for all $t \prime \prime \geqslant t$,

$$
\rho\left(t^{\prime \prime}\right)=R\left(t^{\prime \prime}, t^{\prime}\right) \rho\left(t^{\prime}\right)
$$

and $R\left(t^{\prime \prime}, t^{\prime}\right) \in \mathscr{U}$. Hence, $Q\left(t^{\prime \prime}, t\right)=Q\left(t^{\prime \prime}, t^{\prime}\right) R\left(t^{\prime}, t^{\prime}\right)$ for alI $t^{\prime \prime} \geqslant t^{\prime} \geqslant t$

(A-2) Motions in (A-I) which are twice differentiable. They can be written in the form:

$$
\frac{\partial}{\partial t} \rho(t)=\eta \eta(t) \rho(t)
$$

The set of these $m n^{\prime} s$ is denoted

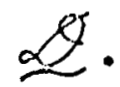


(B-1) Motions such that for all t"l $\geqslant t$,

$$
\rho\left(t^{\prime \prime}\right)=Q\left(t^{\prime \prime}, t^{\prime}\right) \rho\left(t^{\prime}\right)
$$

and $Q\left(t^{\prime \prime}, t^{\prime}\right) \in \mathcal{S}_{I^{\circ}}$

$(B-2)$ Motions in the set $(D-1)$ which are twico differentiable. They can be written in the form

$$
\frac{\partial}{\partial t} \rho(t)=m(t) \rho(t)
$$

The set of these. Mn's is denoted $\mathscr{D}_{\mathrm{I}}$.

$(C-1)$ Motions such that for all $t^{\prime \prime} \geqslant t^{\prime}$,

$$
\begin{aligned}
\rho\left(t^{\prime \prime}\right)=\int_{\left(S U_{N}\right)} d(u) & {\left[P\left(u ; t^{\prime \prime}, t^{\prime}\right) U \rho\left(t^{\prime}\right) U^{+}\right.} \\
& \left.+Q\left(u ; t^{\prime \prime}, t^{\prime}\right) \cup \widetilde{\rho}\left(t^{\prime}\right) U^{+}\right]
\end{aligned}
$$

where the integral is the Haar integral over the unitary uninodular group $S U_{N}$. The probability distribution functions $P\left(u ; t^{\prime \prime}, t !\right)$ and $Q\left(u ; t^{\prime \prime}, t^{\prime}\right)$ satisfy

$$
\begin{aligned}
& P\left(u ; t^{\prime \prime}, t^{\prime}\right) \geqslant 0 ; \quad Q\left(u ; t^{\prime \prime}, t^{\prime}\right) \geqslant 0 \\
& \int_{\left(S U_{N}\right)} d(u)\left[P\left(u ; t^{\prime \prime}, t^{\prime}\right)+Q\left(u ; t^{\prime \prime}, t^{\prime}\right)\right]=1
\end{aligned}
$$

They need not be continuous, but may have delta-function type singnzlaritios on $\mathrm{SU}_{\mathrm{I}}$. 
(C-2) The subclass of motions in the set $(C-1)$ such that for all $t^{\prime \prime} \geqslant t$,

$$
\begin{gathered}
\rho\left(t^{\prime \prime}\right)=\int_{\left(S U_{N}\right)} d(u) P\left(u ; t^{\prime \prime}, t^{\prime}\right) \cup \rho\left(t^{\prime}\right) U^{+} \\
P\left(u ; t^{\prime \prime}, t^{\prime}\right) \geqslant 0 ; \quad \int_{\left(s U_{N}\right)} d(u) P\left(u ; t^{\prime \prime}, t^{\prime}\right)=I
\end{gathered}
$$

(c-3) The subclass of motions in $(c-2)$ such that for all $t " \geqslant t i$,

$$
\begin{array}{cc}
\dot{\rho}\left(t^{\prime \prime}\right)=\int_{(g)} d(g) P\left(g ; t^{\prime \prime}, t^{\prime}\right) U(g) \rho\left(t^{\prime}\right) U^{+}(g) \\
P\left(E ; t^{\prime \prime}, t^{\prime}\right) \geqslant 0 ; & \int_{(\xi)} d(g) P\left(g ; t^{\prime \prime}, t^{\prime}\right)=i
\end{array}
$$

where $\mathcal{G}$ is any compact finite dimensional lie group, and $U(g)$ the representation of elements of $G$ on $\underset{V}{\sim}$ by unitary matrices.

Motions of type (G), especially those in the sets $(C-2)$ and $(C-3)$, are the ones which can be put into the diffusion interpretation discuissed in the Introduction. Motions of type (B) include the most general lincar motions which map $S_{d}$ into $\mathcal{S}_{d}$ and winch preserve the unitorm ensemble, hence the set $(B-1)$ includes notions of type (C). Motions of type (A)

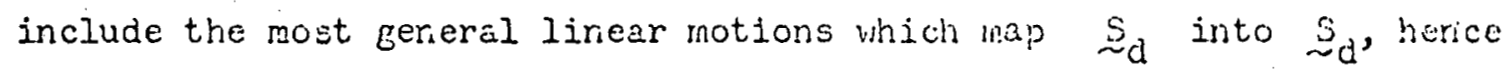
the set $(A-1)$ includes all the other sets, inciuding all notions of type (B) and (C).

Each type of motions will be called stationary if the functions $P\left(g ; t^{\prime \prime} ; t^{\prime}\right), Q\left(u ; t^{\prime \prime}, t^{\prime}\right), P\left(u ; t^{\prime}, t^{\prime}\right)$, or $R\left(t^{\prime \prime}, t^{\prime}\right)$ depend on $\left(t^{\prime \prime}-t^{\prime}\right)$ only. In this case the motions in $(A-2)$ and $(B-2)$ will have constant watrices $M 2$.

If the distribution functions for motions of type (c) are differen- 
tiable, and furthermore satisfy: $P\left(g ; t^{\prime \prime}, t^{\prime}\right)=P\left(g^{-1} ; t^{\prime \prime}, t^{\prime}\right)$, or correspondingly $P\left(u ; t^{\prime \prime}, t^{\prime}\right)=P\left(u^{-1} ; t^{\prime \prime}, t^{\prime}\right)$, and $Q\left(u ; t^{\prime \prime}, t^{\prime}\right)=Q\left(u^{-1} ; t^{\prime \prime}, t^{\prime}\right)$, tlien these motions are included in the set (B-2) with smmetric $M$ 's, It is easy to see that each of the sets forms a semi-group; and each of the sets of non-stationary motions is a convex set. The sets $\mathscr{E}$ and $\mathscr{X}_{\text {I }}$ can be shown to be convex cones.

Finally, we wish to mention briefly an alternative way of writing the equations for motions of type (A) or (B), which may be more natural in some contexts (section $V)$ :

Let $\left\{B_{\alpha}\right\}$ be a fixed orthonormal herratean basis for $y$, and let $\Gamma$ be a linear mapping of $\mathbb{L}$ onto $N$ defined by

$$
[\Gamma(n)]_{\alpha \beta}=\sum_{\gamma, \delta=1}^{N^{2}} n_{\gamma \delta} \operatorname{Tr}\left(B_{\alpha} B_{\gamma} B_{\beta} B_{\delta}\right)
$$

for all $n \in \mathscr{N}$. It can be shown ${ }^{11}$ that

$$
\Gamma(\Gamma(n))=n
$$

It is thus an involution, and accordingly one-to-one. This transformation performed on the matrices $M$ or $\mathbb{R}$ in the equations (III-1) to (III- 4 ) will give equivalent equations of a different form. Thus, for example, if one defines

$$
\mathscr{L}=\Gamma(m)
$$

11. For the proof of Eq. (III-13) as well as Eq. (III-15), see Appendix ] 
then equation (III-2) or (III-4) will take the form ${ }^{11}$

$$
\frac{\partial}{\partial t} \rho(t)=\sum_{\alpha, \beta=1}^{N^{2}} \mathcal{L}_{\alpha \beta}(t) B_{\alpha} \rho(t) B_{\beta}
$$

We will return to this equation later.

B. Conditions for the sets $\mathscr{\perp}, \mathbb{Z}_{I^{\prime}} \mathbb{Z}, \mathbb{Z}_{I}$.

We now study the conditions to be satisfied by an elenent of $\mathcal{L V}$

in order to belong to any of the sets: $\&, \&$, $\mathscr{D}, \mathscr{D}$.

The condition that the hermiticity of density matrices be preserved;

1.e., that $\mathrm{V}_{\mathrm{h}}$ is to be mapped into itself, gives, in any orthonorial herinitean basis,

$$
R^{*}=R
$$

This corresponds to

$$
m^{*}=m
$$

or,

$$
\mathcal{L}^{+}=\mathscr{L} \cdot \quad \text { (where } \mathcal{L}=\Gamma(m) \text { ) }
$$

If the expansion of I into a complete orthonornal hermitean basis is given by

$$
I=\sum_{\alpha=1}^{N^{2}} e_{\alpha} B_{\alpha}
$$


the condition for the preservation of the trace of matrices in $\underset{\sim}{\vee}$ reads,

$$
\begin{aligned}
& \sum_{\alpha=1}^{N^{2}} e_{\alpha} \alpha_{\alpha \beta}=e_{\beta} \\
& \sum_{\alpha=1}^{N^{2}} e_{\alpha} m_{\alpha \beta}=0 \\
& \sum_{\alpha, \beta=1}^{N^{2}} \mathscr{L}_{\alpha \beta} B_{\beta} B_{\alpha}=0
\end{aligned}
$$

Likewise; the condition for the preservation of $I$, needed for the gets $\mathscr{H}_{I}$ and $\mathcal{O}_{I}$; is

$$
\begin{aligned}
& \sum_{\beta=1}^{N^{2}} R_{\alpha \beta \beta} e_{\beta}=a_{\alpha} \\
& \sum_{\beta=1}^{N^{2}} 7 m_{\alpha \beta \beta}=0 \\
& \sum_{\alpha, \beta=1}^{N^{2}} \mathcal{L}_{\alpha \beta}{ }_{\alpha \beta \beta} B_{\beta}=0
\end{aligned}
$$

The condition of mapping $\mathfrak{\sim}_{+}$into 1 tself is soriewhat more complicated. If $E^{\prime}$ and $E^{\prime \prime}$ are any two prinitive hermitean idenpotents, the condition can be written:

$$
\begin{gathered}
\operatorname{Tr}\left(E^{\prime} Q E^{\prime \prime}\right) \geqslant 0 \text { for all } E^{\prime} \text {, E' } \\
\operatorname{Tr}\left(E^{\prime} \cap M E^{\prime \prime}\right) \geqslant 0 \\
\sum_{\alpha, \beta=1}^{N^{2}} \alpha_{\alpha \beta}^{2} \operatorname{Tr}\left(E^{\prime} B_{\alpha} E^{\prime \prime} B_{\beta}\right) \geqslant 0
\end{gathered}
$$


It is not difficult to verify that the conditions (III-16), (III-18), (III-19) and (III-20) are both necessary and sufficient. The conditions (III-18) and (III-19) are simple linear constraints. The condition (III-20) is, however, unfortunately not very explicit in terms of, say; the eigenvalues of the natrices $\mathscr{R}, \mathcal{M}$, or $\mathscr{L}$. They are, of course, related somewhat, and it can be shom ${ }^{12}$ that for rnatrices satistying the conditions.(III-16), (III-18) and (III-19):

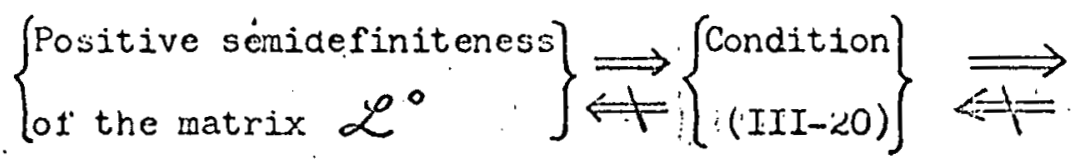

)

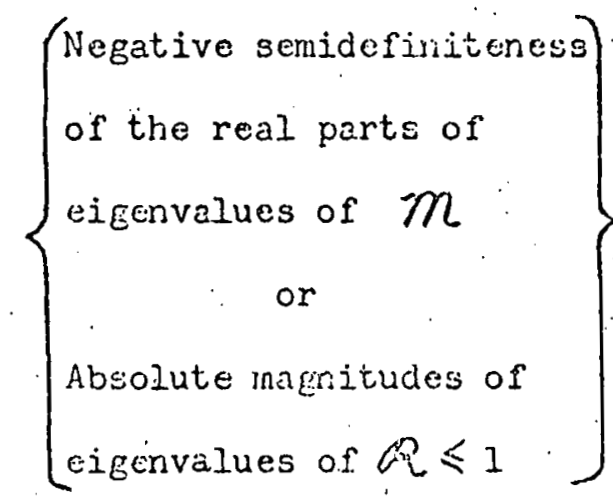

$(I I I-21)$

where $\mathscr{L}^{0}$ is the submatrix of $\mathscr{L}$ which acts on the subspace of $\mathbb{Z}$ of traceless matrices.

C. The Disordering Nature of Motions of type (B). Using tre conditions established in the last. section, we cen now prove a theorem which will partially explain the leeson for our givirg 
the set $\mathscr{Q}_{I}$ special attention.

Theorem: For all motions in the set (B-I), $\rho\left(t^{\prime \prime}\right) \leqslant \rho\left(t^{\prime}\right)$ for a11 $t^{\prime \prime} \geqslant t^{\prime}$.

As was mentioned in section III-P, the set (E-...) includes al.I the motions of type (B) and (C). So in other words the theorem states that. for all motions which transform density matrices tu utrulty ratrices", and for which the uniform ensemble is stable, the time development. can only lead to a decrease in information.

The proof is as follows:

Let the expansion of $\rho\left(t^{\prime \prime}\right)$ into primitive herrittan ideripotents be

$$
O\left(t^{\prime \prime}\right)=\sum_{k=1}^{N} r_{k}^{\prime \prime} E_{k}^{\prime \prime}
$$

and the expension of $\rho\left(t^{\prime}\right)$ be

$$
\rho\left(t^{\prime}\right)=\sum_{k=1}^{M} r_{k}^{\prime} E_{k}^{\prime}
$$

Then

$$
r_{k}^{\prime \prime}=\sum_{m=1}^{N} \operatorname{Tr}\left(E_{k}^{\prime \prime} R E_{m}^{\prime}\right) r_{m}^{\prime} \equiv \sum_{m=1}^{N} d_{k n} r_{m}^{\prime}
$$

It follows from Eq. (III-16) throuch Eq. (III-20) that the metrix

$a_{k m}$ is doubly stochastic, hence $\rho\left(t^{\prime \prime}\right) \leqslant \rho\left(t^{\prime}\right)$.

Clearly the converse statement is also truc, thus:

The subset of motions in $(A-I)$ for which, whenever $t^{\prime \prime} \geqslant t$ ',

$$
\operatorname{Tr} f\left(\rho\left(t^{\prime \prime}\right)\right) \leqslant \operatorname{Tr} f\left(\rho\left(t^{\prime}\right)\right)
$$


for all $f(x) \in F_{c}$, and for every initiel density matrix $\rho(0)$, is. precisely the set $(\mathrm{B}-1)$.

This explains the importance of the motions in tine set (B-1), and

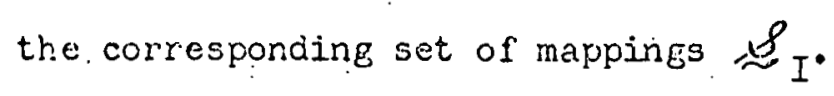

One may note that for motions of type (C), if the distribution functions are nonvanishing in any neighborhood of a point on the group manifold, then one has the stronger result $\rho\left(t^{\prime \prime}\right)<\rho\left(t^{\prime}\right)$, as long. as $\rho\left(t^{\prime}\right) \neq I / N$.

D. Stable Ensembles

The theorem in the last section shows the importance of the stable ensembles in determining the nature of the motion. We wish to elaborate somewhat on some facts about the nature of stable ensembles. (a) Let $Q \mathcal{R} \in \mathcal{O}_{I}$, and $\mathscr{Q} \mathrm{X}=\mathrm{X}, \mathrm{X} \in \mathrm{V}_{\mathrm{h}}$. Let the expansion of $\mathrm{X}$ into hermitean idempotents be given by $X=\sum_{k} x_{k}^{\prime} F_{k}$, where $F_{k}$ correspond to distinct eigenvalues of $x$. Then for every $k$,

$$
R F_{k}=F_{k}
$$

The proof vill be given in Appendix 3.

As a corollary, let $M \in \mathcal{S}_{I}, \quad M X=0, X \leqslant \underline{V}_{h}$. Let $F_{k}$ be defined as above, then for every $k$,

$$
m \mathrm{~F}_{\mathrm{k}}=0
$$

(b) Let $R \in \mathcal{P}_{I}$. Then $\mathscr{Q}$ and $\widehat{Q}$ have the same stable ensembles. 
This follows from a result. of Riesz-Sz. $-\mathrm{Nagy}$, which states thi:t the invariant elements of a contraction on a Hjlbert space coincide with those of the adjoint contraction. ${ }^{13}$ Thet mappines in $\mathcal{B}_{I}$ are contractions will be demonstrated in section IV.

(c) Let $Q \in O B$, and $Q y=x, X \in \mathbb{V}_{\mathrm{h}}$. Expanding $\mathrm{X}$ into prinitive hermitean icicrapotents: $x=\sum_{k=1}^{n} k_{k} \sum_{k}$, where $x_{k} \geqslant 0$ for $1 \leqslant k \leqslant p$, and $x_{k}<0$ for $p<k \leqslant n$.

Let

$$
P \equiv \sum_{k=1}^{p} x_{k} F_{k} ; \quad N \equiv \sum_{k=0+1}^{n} x_{k} E_{k}
$$

Then

$$
Q P=P ; \cdot R N^{-}=N
$$

line proof will also be given in spperdix 3.

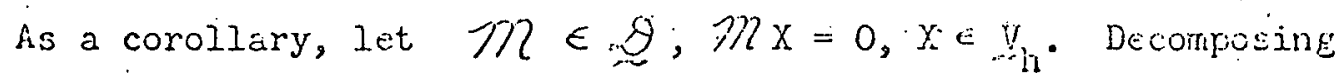
$X$ as above, then

$$
m 2 \mathrm{P}=m 2 \mathrm{~N}=0
$$

(d) For any $q \in \mathbb{U}$, there always exists a density mutrix $\rho_{\mathfrak{s}} \approx \underline{\Xi}_{d}$, such that

$$
R \rho_{s}=\rho_{s}
$$

This follows from the fact that $I$ is a left eigenvector of a wh

13. F. Riesz and B. Sz.-NaE, Acta Sci. Weth. Szoged, 10, 202 (199). 
eigenvalue 1, so there muṣt exist a right eigenvector with eigenvalue equal to 1. Since this eigenvalus is real, the eigenvector may be chosen to be hermitcan. If this eigenvector is either non-negative definite or non-positive definite, we can normalize it to becone a density matrix. If it is not, one can use the result of the last. section, and obtain at least two stable density matrices.

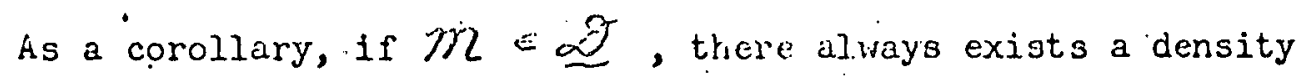
matrix. $\rho_{s} \cdot$ such that

$$
72 \rho_{3}=0
$$




\section{PRCPEFTIES OF STOCHASTIC. MOTIONS. WHICH PRESERVE I}

We have seen in the last section the importance of the set of motions in (B-I): they are the set of all linear notions which take density matrices to density matrices, and wish lead to a decrease in information (or at least not to an increase in informetion) in general. 'lhis set includes all the motione of lype (B) adid (C). On lie uther" nand, motions of type (C) are the ones which neturally allow a diffusion interpretation. It is interesting, therefore, to ask whether the set of motions $(C-1)$ is in fect equal to the set $(B-1)$. We shall show that this is not the case. But first we wish to estiblish sone properties of mappings in the sot $E_{I}$, to wich motions of type (B) correspond, and then return to this question of the relation between the set of". motions (B-I) and the set of motions ( $C-1$ ) in the last subeetion.

A. Unitary and Antiunitary Transformations on $\underset{\sim}{V}$.

Since motions in the set (C-1) arise from conver cominations of unitary and antiunitary transformations on $\underset{V}{V}$, we will iirst study the elenents of 19 which correspond to these trarisformations.

(a) It is easy to see that unitary and antiunitary transfornetions on. $\mathrm{V}$ induce real orthogonal transfomtions in $/ 1$. The set of trose. transformations, which we will denote by $U$, is in general not etgal. to the set of all real orthoconal transiurnations in the will derote the real orthogonal tansformations in ty corresponding to unitary transformations on $\mathrm{V}$ by $Q$, and those corresponding to antiunitiry transifornations by $Q J$, where $j$ is derired by: 


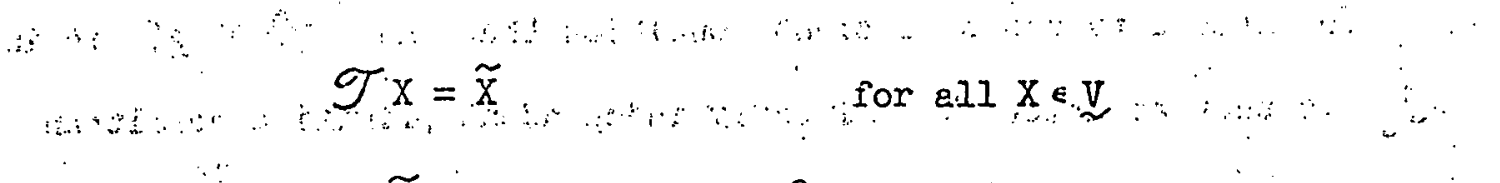

$$
\begin{aligned}
& \text { Since } " \tilde{F}=\mathscr{T} ; \because \because \quad \mathscr{J}^{2}=\mathscr{I}(\text { identity } \ln \mathscr{W})
\end{aligned}
$$

(b) Lot" $R \in \mathcal{E}_{\mathrm{I}}$, and $f(x) \in F_{\mathrm{c}}$. If $R$ is such that for every $\rho \in S_{\mathrm{d}}$

$$
\operatorname{Tr}(\rho)=\operatorname{Tr} f(Q \rho)
$$

then either

or

$$
\begin{aligned}
\mathbb{R} X=U \times U^{+} & \text {for every } x \in \underline{V} \\
R x=U \widetilde{X} U^{+} & \text {for every } x \in V
\end{aligned}
$$

where $U$ is unitary.

$$
\begin{aligned}
& \text { We will sketch the proof. Since } x^{2} \in F_{c} \text {, } \\
& \operatorname{Tr}(\mathscr{X}, \mathscr{R Y})=\operatorname{Tr}(X Y) \text { for any } X, Y \in V_{h}
\end{aligned}
$$

Together with the fact that $\mathcal{R}$ takos primitive hermitean idempotents into primitive hermitean idempotents ( $\mathrm{Eq}$. (II-I8)); and using Wigner's theoren about transformations in quantur mechanics, one has the result.

14. Let there be given an invertible tranisformation $\mathrm{T}$ on all rays of a Hilbert space such that if $\phi_{1}$ and $\phi_{2}$ are unit vectors contained in the arbitrary rays $R_{1}$ and $R_{2}$, respectively, and $\phi_{1}^{T}$ and $\phi_{2}^{T}$ are unit vectors contained in the corresponding transformed rays $R_{1} T$ and $R_{2} T$, respectively, then $\left|\left(\phi_{1}, \phi_{2}\right)\right|=\left|\left(\phi_{1}{ }^{\mathrm{T}}, \phi_{2}{ }^{\mathrm{T}}\right)\right|$. The theorem then asserts that the ray-transformation $T$ can be considered as being induced by either a unitary transformation or an antiunitary transformation, on all vectors of the Hilbert space. See E.P.Wigner, Group Theory, (Academic Press, New York, 1959), translated by. J.J.Grifin, Chap.20, Appendix, p. 233 . 
(c) The necessary and sufficient condition that any $R \in \mathscr{S}_{I}$ is in $\mathbb{Z}_{\mathrm{U}}$ is that at least. $\mathrm{N}^{2}$ linearly independent primitive hermitean idempotents are taken into prinitive hermitcan idempotents. 15

This statement is readily verified using Eq. (IV-15) of the next section.

B. Norms of Vectors in $\mathrm{V}$ under Mappings in $\mathscr{J}_{I}$.

Let us first consider the mapping of a sphere in an n-diriensional real Euclidean space $R^{n}$. Into itself. Let $T$ be any real linear homogeneous mapping on $\stackrel{\mathrm{R}}{ }^{\mathrm{n}}$ satisfying

$$
(T x, T x) \leqslant(x, x) \quad \text { for } a l l x \in \underline{R}^{n}
$$

$T$ is a contraction in the terminology of reference 13.

If $x_{1}, x_{2}, \ldots, x_{m}^{x}(m \leqslant n)$ is a set of: w linearly independent vectors such that

$$
\left(T_{x_{k}}, T_{x_{k}}\right)=\left(x_{k}, x_{k}\right) \quad \ldots k=1,2, \ldots, m
$$

then, denoting the subspace spanned by $x_{I}, x_{2}, \ldots, x_{-n}$ by $V_{n n}$, it is easily verified that, for all $y<\underline{R}^{n}$ and $x \in \underline{V}_{\mathrm{V}}$,

$$
\left(T_{x}, T_{y}\right)=(x, y)
$$

We will now show that mappings in ${ }_{I}$ are contractione; i.e.s for 15. For more necossary and sufîcicnt conditions, see ref. 5. 
any. $R \in, \mathscr{Q}_{\mathrm{I}}$ and: $\mathrm{x} \in \mathrm{V}_{\mathrm{h}}$, we have

$$
\operatorname{Tr}(R x, R x) \leqslant \operatorname{Tr}(X X)
$$

This statement trivially holds for density matrices since $x^{2}=F_{c}$. For arbitrary $\mathrm{X}<\mathrm{y}_{\mathrm{h}}$, one can construct a density matrix $\rho=(I-\delta) I+\epsilon \times \mathrm{X}$ with suitable srnall constants $\delta$ and $\varepsilon$; then Eq. (IV-10) follows.

We can now apply Eq.:(IV-9), and obtain the follorring conclusions:

(a) If $E^{\prime}$ and $E^{\prime \prime}$ are primitive hermitean idempotents satisfying $E^{\prime} E^{\prime \prime}=0$, then

$$
\left(Q E^{\prime}\right)\left(Q E^{\prime \prime}\right)=0
$$

(b) Let $R \in \mathscr{\ell}_{I}$, and $x \in \underline{V}_{\mathrm{h}}$; if

$$
\operatorname{Tr}(\mathscr{X}, \mathscr{R} x)=\operatorname{Tr}\left(\mathrm{x}^{2}\right)
$$

then for any $Y \in \mathbb{V}_{\mathrm{h}}$,

$$
\operatorname{Tr}(\mathbb{X}, \mathbb{Q} Y)=\operatorname{Tr}(X Y)
$$

(c) For a fixed $R \in \mathscr{E}_{I}$, the set of all $x \in \underline{V}_{h}$ for which

$$
\operatorname{Tr}(R x, Q x) \cdot=\operatorname{Tr}\left(x^{2}\right)
$$

form a subspace. We denote this subspace by $\underline{V}_{0}$. For any $Y \in V_{\mathrm{li}}$ and $\mathrm{X} \in \mathrm{V}_{R}$

$$
\operatorname{Tr}(R X, R Y)=\operatorname{Tr}(X Y)
$$


(d) If $X \in \mathrm{V}_{\mathfrak{K}}$, so is $x^{\mathrm{n}}$ for any positive integer $n$.

(e) If $X \in V_{R}$, then $X$ and $R X$ are unitary or antiunitary equivalent. (The unitary or antiunitary transforintion in general depends on $\mathrm{X.})$

The statement (d) follows trom $\operatorname{Tr}(X-C R Q R)^{2}=0$ for $X \in \underline{V}_{h}$, and Lq. (IIL-26); the other statements are easy to verify and will not be demonstrated.

c. Properties of $\mathscr{S}_{I}$ as a Convex Set.

(a) Boundory and Inition Pojnts

It is not difficlilt to show that the interior points of $I$

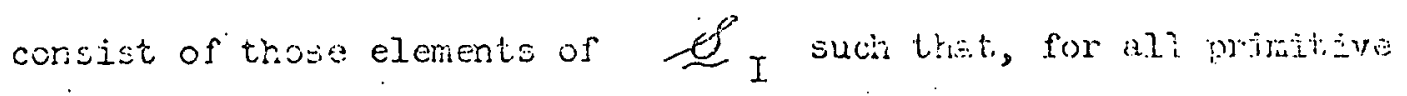
hermitean idempotents $E^{\prime}$ and $E^{\prime \prime}$, and a rixed $\diamond$,

$$
\operatorname{Ir}\left(E^{\prime} R E^{\prime \prime}\right) \geqslant \delta>0
$$

The boundary consists of elements of fuch that for at least one pair of primitive hermitear idempotents $\mathrm{B}^{\prime}$ and $\mathrm{E}^{\prime \prime}$,

$$
\operatorname{Tr}\left(E^{\prime} \not E_{E^{\prime \prime}}\right)=0
$$

(b) Extreme Points

Before ve proced we wish to revien some well-hmom romits and oovver sets in connection with their extrere points: 
(i) A closed, bounded, convex set is the convex hull of its extreme points. 16

(ii) Every point of a closed, bounded, convex set in $\mathrm{R}^{\mathrm{n}}$ is a convox combination of not more than $(n+1)$ extreme points. 17

The convex set $\mathcal{O}_{I}$ is clearly closed and bounded; and these theorens thus show the importance of knowing its extreme elements, Now the elements in $\mathscr{S}_{\mathrm{U}}$ are obviously extreme points of $\mathscr{S}_{\mathrm{I}}$; it is equally obvious that, although they also are extreme points of the convex set $\mathcal{E}$ (see,e.e.; reference 5), they do not forn a.l. the extrerre points of 4 . The nontrivial question is whether they do form all the extreme points of ' If this wore tmie, then by the theorera' $(i)$ above, one would be able to establish the equivalence of the set of motions (B-I) and the set of motions (c-I). Furthernore, from the theorern (ii) above, one would bo able to conclude that every mpoing in $\mathscr{E}_{I}$ can be expressed as a convex combination of a finite number of unitary and antiunitary transformations on $\mathrm{V}$. We will show that for $\mathrm{N}=2$, the set $f_{U}$ indeed form all the extremo points of $\mathscr{S}_{\mathrm{I}}$

In this case, all the real ortlogonal transformations in 1 , subject. to the conditions (III-IEa) and (III-IGa), belong to the thisec dimensional real orthogonal group, which in turn is the romomorphic imulge of the 2-dimensional unitary unirodular eroup. This is trost easily secm by considering the form of matrices in th satisfying Eq. (III-1Eo) ard (III-I ia) for a special basis $\left\{B_{a}\right\}$, such that $B_{1}=(N)^{-\frac{1}{2}}$ I, and all the other $B_{\alpha}$ 's are traceless. These matricos $O$ will have the 16. Reference 4, p. 38, Ex. 2 .

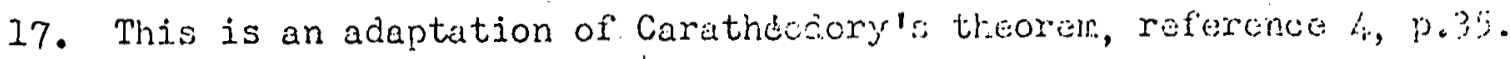


following form in such a basis:

$$
Q_{I I}=I ; \quad R_{I \alpha^{\prime}}=R_{a^{\prime} I}=0 \quad\left(a^{\prime}=2,3, \ldots, N^{2}\right)(I V-I 8)
$$

This holds for any $N_{0}$ For the particular case of $N=2$, the subratrix $Q_{a^{\prime} \beta^{\prime}}\left(\alpha^{\prime}, \beta^{\prime} \neq 1\right)$ acts on the three dimensional subspace of $V$ of traceless matrices; and, from the correspondence to the two dimensional unitary uninodular group mentioned above, all real orthogonal transformaticns in $7 /$ satisfyirg conditions. (III-18a) and (III-19a) wiIl be in $\mathbb{Q}_{\text {U. }}$

Now for any $\mathfrak{R} \in \mathbb{\&}$, we can wite, by polar decomposition,

$$
R=\cos Q
$$

such that $Q$, is real orthoconal and $\mathscr{g}^{\circ}$ is real, symetric, and positive semidefinite, with both $Q$ and gotisfying (III-ISii) and (III-I9a). Since $Q \in \mathbb{E}_{U}, y j \in \mathbb{S}_{I}$ and has eifenvalizes (which are real) not exceeding unity in magnitude. clearly ox is in the conver hull of $\mathscr{H}$; conseruently $\mathscr{Q}$ is also.

ons concludes therefore that the set of motions (B-l) is cemoletoly eauivelent to the set ef motions (c-1) for the cess $1=2$. We wish to point cut that although the motions (C-2) more natwinly fit into the diffusion interpretation, the antiunitary part of the transfornaticna ion motions in $(C-1)$ connot be excluded for physical motions on the rasis of continuity arguments. One may consider, for examplo, the following motions:

$$
\rho(t)=1 / 2\left(I+p_{1} \sigma_{1}+p_{2} \sigma_{2}+p_{3} e^{-t} \sigma_{3}\right)=e^{t / m} \rho(0) \quad(I-20)
$$


where $|\underset{\sim}{p}| \leqslant 1$, and the o's are the Pauli spin matrices. This motion is continuous, but does not bolong in tho set $(C-2)$, as can be verified. So if the antiunitary part is to be excluded at all, this can only be done as a necessary physical requirement, as discussed in section $I-B(b)$, and not as a requirement arising from continuity arguments.

Finally, we wish to show that in spite of the equivalence of the sets of motions $(B-I)$ and $(C-I)$ for $N=2$, which we have demonstrated above, this equivalence in fact doos not hold in general for ail N's.

Let us consider the mapping defined by:

$$
R x=(N-I)^{-1}[\operatorname{Tr}(x) I-x] . \text { for all } x \in V
$$

This mapping cleariy belongs in $\mathcal{f}_{I}$, and has the property that

$$
\operatorname{Tr}(E R E)=0
$$

for all primitive hermitean idempotents. Now suppose that $\mathcal{R}$ is a convex combination of unitary and antiunitary transformations; then becalise of Eq. (III-20) each unitary transformation in this combination mast satisfy:

$$
\operatorname{Tr}\left(\mathrm{EUEU}^{+}\right)=0 \quad \text { for all. E }
$$

and each antinunitary transformation in the combination satisfies:

$$
\operatorname{Tr}\left(\mathrm{EUEUE}^{+}\right)=0 \text { for } \operatorname{all} \mathrm{E}
$$

It is obvious that there does not exist any unitary transformation $U$ which obeys Eq. (IV-23). On the other hand, if a unitary transformation U 
satisfies Eq. (IV-24), then the trace. of $U$ with any symmetric matrix in $\mathrm{V}$ is zero, and $U$ is skew-symmetric. There are no skew-symmetric nonsingular matrices of odd dimensionality. Hence, unitary and antiunitary transformations on $\mathrm{V}$ cannot form all the extreme points of $\mathbb{f}_{\mathrm{I}}$, at least not for odd $\mathrm{N}>2$. 


\section{THE DIFEUSION EQUATIONS}

\section{A. Derivation of the Diffusion Equations}

We will now return to a rore detailed study of stochastic motions which correspond to some difruston process on the group manifold of a Lie group, i.o., motions of the type (C-3). Then the motions are sufficiently continuous; we can derive, in accordance with tho physical plcture discussed in the Introduction, diffusion equations for the distribution function $\mathrm{P}(g ; t ", t)$, and correspondingly for the denster matriz $\rho(t)$, as woll 00 for the phase space density function $\mathrm{D}(\overline{\mathrm{p}}, \overline{\mathrm{a}}, \mathrm{t})$ of a classical system. The derivation will bo somemat heuristic in nature to emphasize the simplicity of tho underlying jeas. We wish, however, to keen the discussion in a general framework without comnituent to specific grouns or specific representations; thus wo will not be discussine speciric kinds of Browian motions, for instence, but any type of Bromian motion whore the random transformations generate a Lie group, such as the force-free rotational Bromicin motion of rigid bodies ${ }^{1 S}$, will belong in this franeworl.

We vill first derive the diffusion equation for the distribution function: $P(g ; t) \equiv P(g ; t, 0)$. . Consider the change in distribution in 2 time interval $\Delta t$. A point on the group manifold, representing the state of the ensemble, will diffuse in different directions. If the moticin is sufficientiy continuous, the distances moved corienpuring to an infinitesimal $\Delta t$ will be small, i.e., they will correspond to group elonomts in the neighborhood of identity, and hence can be written in the cxponcrtict form $\exp \left(\sum_{\mu=1}^{K} x_{\mu} a_{\mu}\right)$, where $x_{\mu}$ are real paratieters, and $a_{\mu}$ form a kisis

13. See W. H. Furry, Phys. Rev., 107, 7. (1957); and L. D. Farro, Liy. Rev., $279,53 \div(1900)$. 
of $\mathcal{O}$; the Lie algebra associated with the group $\mathscr{G},(\mu=1,2, \ldots, \mathrm{K}$, $\mathrm{K}=$ dimensionality of $\mathcal{F})$. The weighting function for the probabilities. of various increments will bo denoted $w\left(x_{1}, x_{2}, \ldots, x_{K} ; \Delta t, t\right)$, or simply $w(x ; \Delta t, t)$. He then have, according to what was said, 19,20

$$
P(g ; t+\Delta t)=\int d x_{1} \ldots \int d x_{K} w(x ; \Delta t ; t) P\left(e^{-x \mu a \mu} g ; t\right)
$$

The function $w(x ; \Delta t, t)$ is of course real and positive, and satisties.

$$
\int d x_{1} \ldots \int d x_{K} w(x ; \Delta t, t)=1
$$

We further impose the continuity of the rotion as requidoments on $w(x ; \Delta t, t)$ and $P(g ; t):$

$$
\operatorname{Lim}_{\Delta t \rightarrow 0} w(x ; \Delta t, t)=\prod_{\mu=1}^{K} \delta\left(x_{\mu}\right)
$$

(ii) The moments

$$
\dot{\rho}_{\mu}(t)=\operatorname{Iin}_{\Delta t \rightarrow 0} \int d x_{1} \ldots \int d x_{K} w(x ; \Delta t, t) x_{\mu} / \Delta t
$$

and

$$
\mathscr{B}_{\mu \nu}(t)=\frac{1}{2} \operatorname{Lim}_{\Delta t \rightarrow 0} \int d x_{I} \ldots \int d x_{K} v(x ; \Delta t, t) x_{\mu} x_{\nu} / \Delta t
$$

exist; and higher moments are negligible.

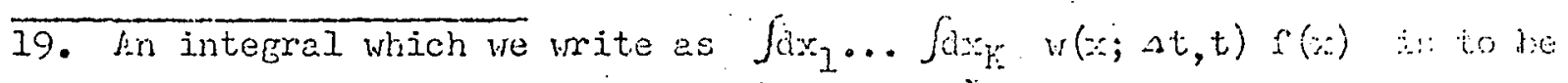

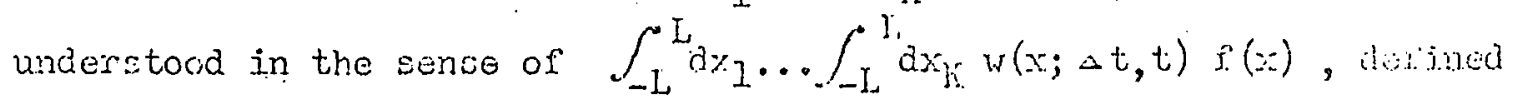
for arbitrary fixed, positive $L$, and $\Delta t \rightarrow 0$.

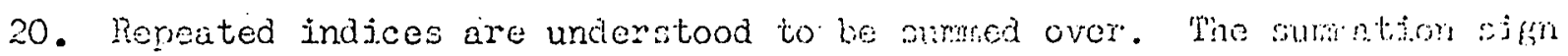
is often onitted where it is clest thet tho range of sumation is over the dirensions of the algebras conecrned. 
(iii) $P\left(\exp \left(x_{\mu} a_{\mu}\right) g ; t\right)$ allows a Taylor expansion in $x_{\mu}$ around $x_{\mu}=0$. With these assumptions, one Imriediately obtains from Eq. (V-I), the differential equation ${ }^{2 I}$ :

$$
\begin{aligned}
\frac{\partial}{\partial t} \mathrm{P}(g ; t)=\mathcal{B}_{\mu}(\mathrm{t}) & {\left[\frac{\partial}{\partial x_{\mu}} \mathrm{P}\left(\operatorname{erp}\left(-\mathrm{x}_{\gamma} a_{\gamma}\right) ; t\right)\right]_{x_{\gamma} \rightarrow 0} } \\
& \left.+\mathscr{G}_{\mu \nu}(t)\left[\frac{\partial^{2}}{\partial x_{\mu} x_{\nu}} \mathrm{P}\left(\exp \left(-x_{\gamma}{ }^{2}\right)\right) ; t\right)\right]_{x_{\gamma} \rightarrow 0}
\end{aligned}
$$

This eoution can be applied to classical as vell as quantur mechanical systems. We vill first apply it to the tirne rate of change of the density matrix of a quanturn mechanical system, by use of Eq. (III-10):

$$
\begin{aligned}
\frac{\partial}{\partial t} \rho(t)= & \int_{(\Theta)} d(g)\left[\hat{O}_{\mu}(t) \frac{\partial}{\partial x_{\mu}} P\left(\exp \left(-x_{\gamma} a_{\gamma}\right) g ; t\right)+\right. \\
& \left.\mathscr{b}_{\mu \nu}(t) \frac{\partial^{2}}{\partial x_{\mu} x_{\nu}} P\left(\exp \left(-x_{\gamma}{ }^{a} \gamma\right) g ; t\right)\right]_{x_{\gamma}=0} U(g) \rho(0) U^{+}(g)
\end{aligned}
$$

Using the invariance of the group measure under. left translation, and denoting the representation of a as stew-hermitean operators in $\square_{h}$ by $-1 A_{Y}$, one obtains

$$
\begin{aligned}
& \frac{\partial}{\partial t} \rho(\mathrm{t})=\int_{(\mathscr{G})} \mathrm{d}(\mathrm{g}) \mathrm{P}(\mathrm{g} ; \dot{\mathrm{i}})\left\{\left[\mathcal{B}_{\mu}(\mathrm{t}) \frac{\partial}{\partial x_{\mu}}+\mathscr{B}_{\mu \nu}(\mathrm{t}){\frac{\partial}{\partial x_{\mu} x_{\nu}}}_{\mu}\right] \mathrm{x}\right. \\
& \left.\exp \left(-i x_{\gamma} p_{\gamma}\right) U(g) \rho(0) U^{+}(g) \exp \left(i x_{\gamma}{ }^{A} \gamma\right)\right\}_{\gamma}=0
\end{aligned}
$$

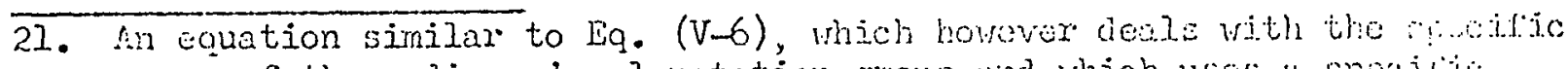
group of three dimensional rotation group win wich uses a onodict representation, has been obtained by $1 . \mathrm{H}$. Hury, ret. $2:$. 
or,

$\frac{\partial}{\partial t} \rho(t)=-i \beta_{\mu}(t)\left[A_{\mu}, \rho(t)\right]-\mathscr{E}_{\mu \nu}(t)\left[A_{\mu},\left[A_{\nu}, \rho(t)\right]\right]$

The motion is therefore seen to consist of separately a unitary. transformation, represented by the first term on the right, and a diffusion represented by the second term. Since we wish to concentrate on studying the diffusive part of the motion, we will not consider the first term, assuming eitrer that $w(x ; \Delta t, t)=w(-x ; \Delta t, t)$, so that $\mathscr{B}_{\mu}(t)=0$, or that the unitary motion is absorbed by transforming to an "interaction representation" 22

We vill next apply the equation $(V-6)$, without the unitary term, to tho phase space censity function of a classical systom, and will simpify. the notation somewhat by miting:

$$
\left[\frac{\partial}{\partial x_{\mu}} P\left(\exp \left(-x_{\gamma} a_{\gamma}\right) g ; t\right)\right]_{x_{\gamma}-0} \equiv \hat{a}_{\mu} P(g ; t)
$$

Let us denote the phase spece density by $D\left(\bar{p}_{I}, \overline{\mathrm{p}}_{2}, \ldots, \overline{\mathrm{p}}_{\mathrm{N}} ; \overline{\mathrm{q}}_{1}, \overline{\mathrm{a}}_{2}, \ldots, \overline{\mathrm{q}}_{1 \mathrm{~N}} ; t\right)$; or simply $D(\overline{\mathrm{p}}, \overline{\mathrm{C}}, t)$. For a Hamiltonian tine development, becauwe of the

22. Let us detine, for contint, $\mathscr{S}_{\gamma}$,

$$
\begin{aligned}
& \check{A}_{\mu}(t) \equiv \exp \left(i \hat{S}_{\gamma} A_{\gamma} t\right) A_{\mu} \exp \left(-i g_{\gamma} A_{\gamma} t\right) \\
& \check{\rho}(t) \equiv \exp \left(i \dot{B}_{\gamma} A_{Y} t\right) \rho(t) \exp \left(-i g_{\gamma} A_{Y} t\right) .
\end{aligned}
$$

then

$$
\frac{\partial}{\partial t} \stackrel{v}{\rho}(t)=-\mathscr{b}_{\mu \nu}(t)\left[\check{A}_{\mu}^{V}(t),\left[\check{A}_{\nu}(t), \dot{\rho}(t)\right]\right]
$$

But $\check{A}_{\mu}(t)$ is expressible as a linear sum of the ${ }_{\gamma} \gamma^{1}$ s; henee

$$
\frac{\partial}{\partial t} \check{\rho}(t)=-\mathscr{D}_{\mu \nu}(t)\left[A_{\mu},\left[A_{\nu}, \check{\rho}(t)\right]\right]
$$

Things are more complicated if $\hat{S}_{Y}(t)$ are tine dependent, but the same can in principle be achieved If one knows the solution to the unitary part of the motion. 
invariance of the phase space volume under canonical transformations $p=p(\bar{p}, \bar{q}), q=q(\vec{p}, \vec{q})$, the density at any time is related to the original density $D_{0}(p, q)$ by

$$
D(\ddot{p}, \bar{q}, t)=D_{0}(p=p(\bar{p}, \bar{q}, t), q=q(\bar{p}, \dddot{q}, t)
$$

We again consider the case whero the canonical transfomations cenerat? a finite dimonsional Lie group $\mathcal{E}$, and denote

$$
p^{\left(\varepsilon^{\prime}\right)}(0, i)=p^{\prime} ; \quad p^{\left(g^{\prime \prime}\right)}\left(\bar{p}, a^{\prime}\right)=p^{\prime \prime}
$$

where

$$
\xi^{\prime}, \varepsilon^{\prime \prime} \in \varrho^{\prime} ; \quad \text { and } \varepsilon^{\prime \prime}=\exp \left(-x_{\gamma^{a}} \gamma^{\prime} \mathrm{g}^{\prime}\right.
$$

As $x_{y} \rightarrow 0$, one knows from the theory of inrinftesimal contact transformations that there exist cenerating function it such thet

$$
\begin{aligned}
& p_{i}^{\prime \prime}=p_{i}^{\prime}-x_{\mu} \frac{\partial \mu_{\mu}^{\prime}}{\partial q_{i}} \\
& q_{i}^{\prime \prime}=q_{i}^{\prime}+x_{\mu} \frac{\partial \bar{A}_{\mu}}{\partial p_{i}^{\prime}}
\end{aligned}
$$

Hence

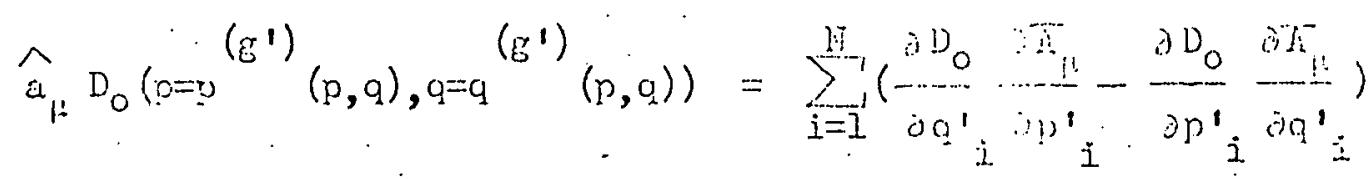

$$
\begin{aligned}
& =\left[D_{0}, \bar{A}_{\mu}\right]
\end{aligned}
$$

where the brackets denote Poisson brackets, and the functions $T_{1}$ ": i...' 
on $g^{\prime}$.

We now consider the diffusion of points on the group manifold, and write the equation which is analogous to Eq. (III-10),

$$
\left.D(p, \bar{q}, t)=\int_{(g)} d(g) P(g ; t) D_{0}(p=p)(\bar{p}, \bar{q}), q=q(g)(\bar{p}, \bar{q})\right)
$$

Applying Eq. (V-6) to this equation, and using the left-invariance of the group rneasure as before, one obtains

$$
\frac{\partial}{\partial t} D(\bar{p}, \bar{q}, t)=\int_{(\mathcal{G})} d(g) P(g ; t) \mathscr{b}_{\mu \nu}(t)\left[\bar{A}_{\mu},\left[\bar{A}_{\nu}, D_{0}\right]\right] .
$$

or, since the Poisson brackets defined with $p^{\prime}, q^{\prime}$ are the same as those defined with $\overline{\mathrm{p}}, \overline{\mathrm{q}}$,

$$
\frac{\partial}{\partial t} D(\bar{p}, \bar{q}, t)=b_{\mu \nu}(t)\left[\bar{A}_{\mu},\left[\bar{A}_{\nu}, D(\bar{p}, \bar{q}, t)\right]\right]
$$

To sumarize, therefore, we have now obtained the rollowing dirfusion equations:

$$
\begin{aligned}
& \frac{\partial}{\partial t} \rho(t)=-\mathscr{C}_{\mu \nu}(t)\left[A_{\mu},\left[A_{\nu}, \rho(t)\right]\right] \\
& \frac{\partial}{\partial t} D(\bar{p}, \bar{q}, t)=\mathscr{C}_{\mu \nu}(t)\left[\bar{A}_{\mu},\left[\bar{A}_{\nu}, D(\bar{p}, \bar{q}, t)\right]\right]
\end{aligned}
$$

and

$$
\frac{\partial}{\partial t} \cdot P(g ; t)=\quad \mathscr{E}_{\mu \nu}(t) \hat{a}_{\mu} \hat{a}_{\nu} P(g ; t)
$$

with

$$
P(g ; 0)=\delta(g)
$$


any suitably defined testing function $P(E)$ on the group manifold,

$$
\int_{(y)} d(g) P(g) \delta\left(g g^{1-1}\right)=P\left(g^{1}\right)
$$

\section{B. Normalization and Integral Identities for $P(g ; t)$.}

The normalization for the distribution function is given by Eq. (III-11). This normalization is consistent with the equation of motion $(\mathrm{V}-22)$, since

$$
\frac{\partial}{\partial t} \int_{(\xi)}^{d(g)} P(g ; t)=-b_{\mu \nu}(t) \int_{(\mathcal{G})} d(g) \hat{a}_{\mu} \hat{a}_{\nu} P(g ; t)=0
$$

For the particulur case where the weighting function $w(x ; \Delta t, t)$ in Eq. $(\mathrm{V} \div \mathrm{I})$ is independent of $t, w(x, \Delta t)$ clearly must agree with $P\left(\exp \left(\mathrm{x}_{\gamma} \mathrm{a}_{\gamma}\right), \Delta t\right)$ aside frorn a factor. In fact, since in this case the choice of the origin of $t$ is arbitrary, the motion being stationary, one would expect from the physical meaning of $P(g ; t)$ that for all $t_{0} \leqslant t$

$$
P(g ; t)=\int_{(\varphi)} d\left(g_{0}\right) P\left(g_{0} ; t_{0}\right) P\left(E g_{0}^{-I} ; t-t_{0}\right)
$$

We will show that this is indeed the case:

If one differentiates the right hand side with respect to $t_{0}$, it is not at first obvious that the derivative is zero. However, the right lind side is clearly a function of $g, t$, and $t_{0}$, and one can write it as $F\left(g ; t, t_{j}\right)$, defined for all $t_{o} \leqslant t$. Since $F\left(g ; t, t_{0}\right)$ satisfies $E q$. (V-22) in $t$, Including the initial condition, as well as the norralization condition, ore concludes from the unicueness of the solution to linear 
differential equations that $F\left(g ; t, t_{0}\right) \cdots$ is independent of $t_{0}$.

$$
F\left(g ; t, t_{0}\right)=F(g ; t)=P(g ; t)
$$

Furthermore, using the independence of $F\left(g ; t_{,} t_{0}\right)$ of $t_{0}$, one may write

$$
\int_{(j)} d\left(g_{0}\right) P\left(g_{0} ; t_{0}\right) P\left(g_{i} i^{-I} ; t-t_{0}\right)=\int_{(j)} d\left(g_{0}\right) P\left(g_{0} ; t-t_{0}\right) P\left(g_{0}^{-1} ; t_{0}\right)
$$

Applying the inversion invariance of Eroup measure ${ }^{23}$ to Eq. $(V-27)$, one obtains

$$
\int_{(g)} d\left(g_{0}\right) P\left(\varepsilon_{0}: t_{0}\right) P\left(g g_{0}^{-1} ; t-t_{0}\right)=\int_{(g)} d\left(g_{0}\right) P\left(g_{0} ; t_{0}\right) P\left(g_{0}^{-1} g ; t-t_{0}\right)
$$

kq. $(y-23)$ can then be used to show that the derivative of the rifht hand side of $\mathrm{Eq}$. (V-25) with respect to $t_{0}$ is indeed zero, thus showing the self-consistency of these relations.

C. Dirfusion Equation and lotiuns of lyoe (ij).

(a) Ue now wish to compare the diffusion equation for the denojity matrix, viz., Eq. (V-20), with the nost generid differentiable notion which takos. density ratrices to density matricos and wich preserves the uniforn? enserable, i.e., motions of: tyme (B-2). We have proviously shorn

23. The Hacr measure is inverse inveriant, as wall as right invorim, il the modular function of the sroup is ecual to one; thics is trie it:

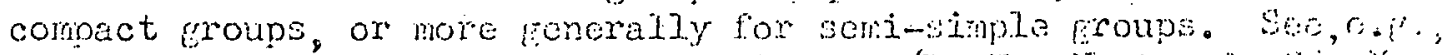

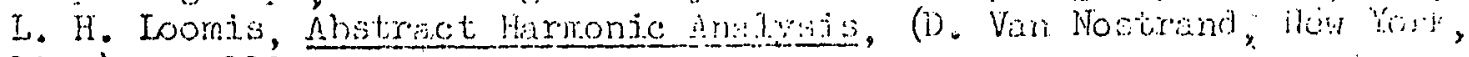
1953), p. 218. 
(section III-A) that the latter motions can be transformed into the form:

$$
\frac{\partial}{\partial t} \rho(t)=\sum_{\alpha, \beta=1}^{N^{2}} \alpha_{\alpha \beta}(t) B_{\alpha} \rho(t) B_{\beta}
$$

If wo now use the conditions (III-18c) and (III-I9c), and denote the symmetric and skew-symnotiric parts of $\mathscr{L}_{\alpha \beta}(t)$ by $\breve{\mathcal{L}}_{\alpha \beta}(t)$ and $\hat{\mathcal{L}}_{\alpha \beta}(t)$, respectively, . . $q$. (V-29) becones,

$\frac{\partial}{\partial t} \rho(t)=-\frac{1}{2} \sum_{\alpha, \beta=1}^{N^{2}} \check{l}_{\alpha \beta}(t)\left[B_{\alpha},\left[B_{\beta}, \rho(t)\right]\right]+$

$$
\sum_{a, \beta=1}^{N^{2}} \hat{\mathscr{L}}_{\alpha \beta}(t) B_{\alpha} \rho(t) B_{\beta}
$$

Thus motions in $(\dot{B}-2)$ can alvays be tmansformed into the form of the diffusion equation $(V-20)$ î $\mathscr{L}_{\alpha \beta}(t)$ is synnetric; which in turn roeans; fromi $\mathrm{Eq}_{1}(\mathrm{III}-12)$ and (III-1/s), that $m(t)$ is symnetric.

As to the part of the notion due to the skew-syrmetric term in Eq. $(v-30)$, which is not includes in the diflusion equation, we can mention brieily some of its properties:

(i) A skew-symotric $\mathscr{L}_{\alpha \beta}$ would give rise to an $7 / 2$ with purely imaginary eigenvalues, which in turn mons thet the solution to Eq. (Tr.-1) will be of an oseillatory nuture, in contrast to tho exponentially deceying nature of the solution correspondine to pure diffusions. (ii) lotions due to skev-simnetic $\hat{\mathscr{L}}_{\alpha \beta}(\mathrm{c})$ alone vill preserve the trace of $\rho^{2}(t)$. Nlso, they take primitive hermitem idomirotants into prinitive heraitesn idempolents.

(iii.) Finslly, it should be nchtioned that motions due to skew-mynnotude $\hat{\mathscr{C}}_{a !}(i)$ alone in eeneral doos not belong in the set (Q-2) besanse of ille inpossibility to meot the condition (III-20). The only possible ckev- 
symmotric $\hat{\mathscr{L}}_{\alpha \beta}(t)$ which by itself (i.e., without an accompanying symmetric part) can satisfy condition (III-20) are the ones which correspond to uni. tary motions.

One concludes, therefore, that the part of motion due to skew-symmetric. $\hat{\mathscr{L}}_{a \beta}(t)$ does not generally lead to a decrease in information.

(b) We wish to make hero a very brief digression. For the density matrix $\because$ which satisfies the diffusion equation $(V-20)$, as for all motions of type (B) and (C), the uniform enserable is a stable final ensemble, though not necessarily the only one. On the other hand, if one modifies Eq. (V-20) slightly by substituting $\left(\rho(t)-\rho_{s}\right)$ for $\rho(t)$ in $\mathrm{Eq}_{1} \cdot(\mathrm{V}-20)$, then clearly $\rho_{\mathrm{s}}$ is a stable rinal ensemble. Furthermore, the rodiricd equation can be written:

$$
\begin{aligned}
& \frac{\partial}{\partial t} \rho(t)=-\mathscr{C}_{\mu ;}(t)\left[A_{\mu} ;\left[A_{;}, \dot{\rho}(t)\right]\right]+ \\
& \left.\mathscr{O}_{\mu \nu}(t)\left[A_{\mu},\left[A_{\nu}, \rho_{s}\right]\right]\right] \operatorname{Tr} \rho(t)
\end{aligned}
$$

so that it is still a homogeneous equation. He will not discuss, however, the relation between $E q .(V-3 I)$ and motions in the set $(A-\dot{2})$. As we have. emphasized, our main concern is with rappines in the set $e_{I}$, and correspondingly with motions of type (B) and (C). 
VI. FURTHER STUDIES RELATED TO

THE DIFIUSION EQUATIONS

\section{A. Definition of Entropies}

Let us define the "entropy" on the group manifold by

$$
s^{\prime}(\dot{t})=-\int_{(g)} d(g) P(E ; t) \ln P(g ; t)
$$

Difforentiating with respect to tine,

$$
\frac{\partial}{\partial t} s^{\prime}(t)=-\int_{(y)} d(g)(\operatorname{Ir} P(g ; t)+I) \cdot b_{\mu \nu}(t) \hat{a}_{\mu} \hat{a}_{\nu} P(g ; t)
$$

or,

$$
\frac{\partial}{\partial t} s^{\prime}(t)=-\mathscr{b}_{\mu \nu}(t) \int_{(y)} d(g) P(g ; t) \hat{a}_{\mu} \hat{a}_{\nu}(\operatorname{In} P(g ; t)+I)
$$

i.e.,

$$
\begin{aligned}
& \frac{\partial}{\partial t} s^{\prime}(t)=\mathscr{O}_{1 \nu}(t) \int_{(\rho)} d(g)\left\{P^{-1}(\xi ; t)\left[\hat{a}_{H} \dot{p}(g ; t)\right]\left[\hat{a}_{\nu} P(g ; t)\right] .\right. \\
& \left.-\hat{a}_{\mu} \hat{a}_{\nu} p(g ; t)\right\}
\end{aligned}
$$

Hence

$$
\frac{\partial}{\partial t} s^{\prime}(t)=\mathscr{b}_{\mu \nu}(t) \int_{(\xi)} d(g) P^{-I}(g ; \dot{\xi})\left[\hat{a}_{\mu} P(\xi ; t)\right]\left[\hat{a}_{\nu} p(g ; t)\right]
$$

Thus the entropy $s^{\prime}(t)$ is a non-decreasing function of time if $\mathscr{G}_{\mu \mu}(i)$ is positive semi-definite.

On the other hand, the entropyr of the cnsemble, as defined by $\mathrm{Eq} \cdot(I I-3)$, is

$$
s(t)=-\operatorname{Tr}(\rho(t) \ln \rho(t))
$$


And it can easily be show that $s(t)$ is also non-decreasing with time if $\mathscr{C}_{\mu \nu}(t)$ is positive semi-definito.

The $\mathscr{b}_{\mu \nu}(t)$ which is obtained from the definition $(V-5)$ is, of course, automatically positive semi-definite. In fact, in this case we know that $s(t)$ is nondecreasing with tine by the theorem (II-10). One may ask whether $s^{\prime}(t)$ is equivalent to $s(t)$ except for a miltiplicative constant which depends on the initial density matrix $\rho(0)$. It is easily seen, however, that this is not the case. That may seem paradoxtai at this point is the apparcint violation of the vell-mom theorem that the definition of entropy is unique . A closer examina- . tion of the assumptions requirea for the uniqueness proof shovs, hoverer, that there is no contradiction. To obtain a unique expression for the entropy, it is necessary to specify the set of motions for which the entropy is to remain constant. In quantum mechenics this set includes a1.1. unitery motions. On the other hand, the most freneral information preserving motions which have here been considered on the rroup manifold are those corresponding to continuous inner automorphisms. This set is too small to permit a unioue determination of the entropy.

To see this more clearly one may conider the Hilbert space of all square integrable functions or the roup mifold. The inner automorphions by no means correspond to the set of a.l.t unitary transformations on this Hilbert space. Therefore the "entropy" $s^{\prime}(t)$ defined by Eq. (VI-I) is not necessarily the physical entropy, but merely a quantity the increase of mich indicates the diffusive nature of the motions.

24. See, for example, A. I. Khinchin, Gthenaticnl Foundatione of Informtion Theory, (Dover Fublication, Nev York, 1957). 
B. Possible Reduction of the Diffusion Equation for $P(g ; t)$.

When the solution of Eq. $(V-22)$ for $P(g ; t)$ can be obtained by solving equations of lower dimensionalities (i.e., fewer variables) but of the same form, and such that the sum of the dimensionalities of these equations does not exceed that of the original equation, we say that the equation is reduced, or the integration problem is reduced. We will give a somewhat trivial example of such a reduction when the group is a direct product of two invariant subgoups.

Let $\mathscr{Y}=\mathscr{Y}^{\prime} O \mathscr{Y}^{\prime}$ Ve denote the lie algebra associated with $\mathcal{G}^{\prime}$ by $\mathscr{S}^{\prime}$, and let ${ }^{\prime}$, form a basis of $\mathscr{M}^{\prime}, \lambda_{\mu}^{\prime}=1,2, \ldots, K^{\prime} ; K^{\prime}=d i m e n-$ sionality of $\left.\mathcal{S}^{\prime}\right)$. The alobira associated with $\mathcal{G}^{\prime \prime}$ is denoted $\mathcal{S}^{\prime \prime}$, of which ${ }^{2} \mu^{n}$ form a basis, $\left(\mu^{\prime \prime}=1,2, \ldots, K^{\prime \prime} ; K^{\prime \prime}=\right.$ dim. of $\left.S^{\prime \prime} ; K^{\prime}+K^{\prime \prime}=K\right)$.

In this case one could expect thet the equation $(V-22)$ can be reduced, since there is no non-commitivity to couple the tro invariont subgoups ' $\mathcal{F}^{\prime}$. and $\mathcal{G}^{\prime \prime}$. Such is, however, not the case, at least not in any obvious way, because the coefficients $f_{\mu \nu}(t)$ still may couple the distributions on the respective manifolds of the subvoups. Such couplings are absent only if all $\hat{E}_{\mu^{\prime \prime} \nu^{\prime}}$ and $\hat{\theta}_{\mu \prime \mu^{\prime \prime}}$ are zero. Under such an assumption, which we will now make, it is easy to see that the equation indeed separates.

For any. $g \in \mathcal{G}$, there is a unicue factorization such that $E=\varepsilon^{\prime} g^{\prime \prime}$, with $g^{\prime} \in \mathcal{G}^{\prime}$, and g't $\mathscr{\vartheta}^{\prime \prime}$.

Let us define:

$$
P(E ; t)=P_{I}\left(g^{\prime} ; t\right) P_{2}\left(E^{\prime \prime} ; t\right)
$$

Since

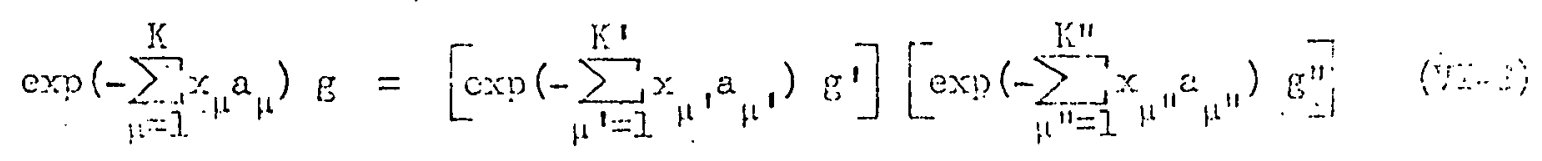


we have,

$$
\hat{a}_{\mu}, \hat{a}_{\nu}, P_{1}\left(g^{\prime} ; t\right) P_{2}\left(g^{\prime \prime} ; t\right)=\left[\hat{a}_{\mu}, P_{1}\left(g^{\prime} ; t\right)\right]\left[\begin{array}{c}
\hat{a} \\
\hat{\nu}^{\prime \prime}
\end{array} P_{2}\left(g^{\prime \prime} ; t\right)\right]
$$

Differentiating Eq. (VI-7) with respect to $t$, one gets

$$
\begin{array}{r}
\frac{\partial}{\partial t} P(g ; t)=\mathscr{O}_{\mu^{\prime} \nu^{\prime}}(t)\left[\hat{a}_{\mu^{\prime}}, \hat{a}_{\nu^{\prime}} P_{I}\left(E^{\prime} ; t\right)\right] P_{2}\left(g^{\prime \prime} ; t\right) \\
+\hat{O}_{\mu^{\prime \prime} \nu^{\prime \prime}}(t) P_{1}\left(g^{\prime} ; t\right) \hat{a}_{\mu^{\prime \prime}} \hat{a}_{\mu^{\prime \prime}} P_{2}\left(g^{\prime \prime} ; t\right)
\end{array}
$$

Dividing through by $P(g ; t)$,

$$
\begin{aligned}
& P_{I}^{-I}\left(g^{\prime} ; t\right)\left\{\frac{\partial}{\partial t} P_{1}\left(g^{\prime} ; t\right)-b_{\mu}^{\prime},(t) \hat{a}_{\mu} \hat{A}_{y}, P_{I}\left(g^{\prime} ; t\right)\right\}=
\end{aligned}
$$

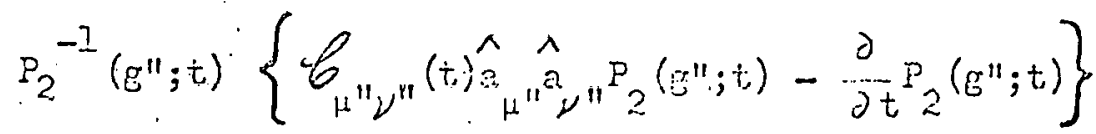

The left hand side deperds on $g^{\prime}$ alone, and the right hand side depends only on $g^{\prime \prime}$; hence

$$
\begin{aligned}
& \frac{\partial}{\partial t} P_{I}\left(g^{\prime} ; t\right)=\mathscr{b}_{\mu^{\prime} \nu^{\prime}}(t) \hat{a}_{\mu} \hat{a}_{\nu^{\prime}} \dot{p}_{I}\left(g^{\prime} ; t\right)+f(t) P_{I}\left(g^{\prime} ; t\right) \\
& \frac{\partial}{\partial t} P_{2}\left(g^{\prime \prime} ; \dot{t}\right)=\mathscr{b}_{\mu^{\prime \prime} \nu^{\prime \prime}}(t) \hat{a}_{\mu^{\prime \prime} \hat{a}^{\prime \prime}{ }^{\prime}} P_{2}\left(g^{\prime \prime} ; t\right)-f(t) \cdot P_{2}\left(g^{\prime \prime} ; t\right)
\end{aligned}
$$

The function $f(t)$ can be set to zero by the recuirencht of conservation of normalization for $P_{1}\left(g^{\prime} ; t\right)$ and $P_{2}\left(\xi^{\prime \prime} ; t\right)$. The initial conijtions consistent with Iq. (V-22) mast be: $P_{I}\left(g^{1} ; 0\right)=\varepsilon\left(\xi^{\prime}\right): P_{2}\left(g^{\prime \prime} ; 0\right)=0\left(q^{\prime \prime}\right) ;$ hence $P_{I}\left(g^{\prime} ; t\right)$ and $P_{2}\left(g^{\prime \prime} ; t\right)$ satisfy equations of the some form as Eq. (V-22), but of lover dimensionalities. 
VII. VOTIOHS OF DFUMICAL VERIABLES

AND SYRTTRIES OF VOTIONS

A. Tire Development of Dimamical Variables.

For sections VII-A and VII-B, we wiIl go beyond motions of type (B) and $(C)$, and consider general motions of type $(A)$ :

$$
\begin{aligned}
& \rho\left(t^{\prime \prime}\right)=\mathscr{R}\left(t^{\prime \prime}, t^{\prime}\right) \rho\left(t^{\prime}\right) \\
& R\left(t^{\prime \prime}, t^{\prime}\right) \in \mathscr{Q} \text { for } a l I t^{\prime \prime} \geqslant t^{\prime}
\end{aligned}
$$

Let us consider the time rate of change of the ensemble jverages of some aynamical variable $\mathrm{K}$.

If we define:

$$
<K>_{a v}(t) \equiv \operatorname{Tr}(\rho(t) K)
$$

then

$$
<K\rangle_{\text {av }}\left(t^{\prime \prime}\right)=\operatorname{Tr}\left[R\left(t^{\prime \prime}, t^{\prime}\right) \rho\left(t^{\prime}\right), K\right]=\operatorname{Tr}\left[\rho\left(t^{\prime}\right), \tilde{R}\left(t^{\prime \prime}, t^{\prime}\right) K\right]
$$

We may therefore simply consider the action of $\tilde{R}\left(t{ }^{\prime \prime}, t^{\prime}\right)$ on $k$, and then average over a fixed initial ensemble. In fact, denotine.

$$
K(t) \equiv \widetilde{R}(t, 0) K
$$

then $K(t)$ satisfies an equation analogous to Eq. (VII-I),

$$
\begin{aligned}
& K\left(t^{\prime \prime}\right)=\widetilde{R}\left(t^{\prime \prime}, t^{\prime}\right) K\left(t^{\prime}\right) \\
& \tilde{R}\left(t^{\prime \prime}, t^{\prime}\right) \in \mathscr{f} \text { for a]l } t^{\prime \prime} \geqslant t^{\prime}
\end{aligned}
$$


If the set of $K(t)$ spans a vector space $V_{K}$ whose dimensionality is smaller than that of $\underline{V}_{h}$, then Eq. (VII-5) is actualiy simpler to deal with than Eq. (VII-1). Such considerations are of practical importance since in actual problems one is often intorested prinarily in the chanco with time of the averages of vectors in $\mathrm{v}_{\mathrm{K}}$ for some important variable $K$, and not so much in the density matrix itself.

For the case whore $\rho(t)$ satisfies the diffusion equation, one has

$$
\frac{\partial}{\partial t}<K>_{a v}(t)=-\mathscr{L}_{\mu \nu}(t) \operatorname{ir}\left\{\rho(t),\left[A_{\nu},\left[A_{i t}, K\right]\right]\right\}
$$

or,

$$
\frac{\partial}{\partial t}<K>_{a v}(t)=-\mathscr{H}_{\mu \nu}(t)<\left[A_{\nu},\left[A_{\mu}, K\right]>_{D}(t)\right.
$$

So any variable $A_{\mu}$, wich commutes with $K$ as well as with $\left[\Lambda_{\mu}, K\right]$." will arop out of the expression. Differently stated, if the group is a product of two normal subgroups, one of which acts like identity on $k$, . then that subgroup can be lefi out of consideration.

Finally, we aention a possible wy of defining "the amourt of iniormation about $K$ possessed for the ensemble $\rho "$ : Inf $\rho(K)$.

Let the expansion of $K$ into idempotents be:

$$
K=\sum_{j=1}^{p} k_{j} F_{j}
$$

where the $F_{j}$ are associated, with the distinct eircnwlues of $K$. Let, $f_{j}=$ Tr $\left(\rho \vec{r}_{j}\right)$; they represent the probabilities thet the systati is in one or the subspaces defined by $F_{j}$. We may then use the measume:

$$
\operatorname{In} \hat{i}_{\rho}(K)=\ln (0)+\sum_{j=1}^{p} \hat{r}_{j} \ln \hat{r}_{j}
$$


The reasure of information so defined has the following properties:

(i) the smallest value assumed by $\operatorname{Inf} \rho(K)$ is 0 , while the largest value assumed is $\ln (p)$; (ii) it $Y=f(K)$ is any function of $K$ such that $f\left(k_{n}\right) \neq f\left(k_{j}\right)$ for $n \neq j$, then $\operatorname{Inf}_{\rho}(Y)=\operatorname{Inf}(K)$.

One notes that for a disordering diffusion process, i.e., $\rho(t)$ tends towards the uniform enserable, Info $(K)$ need not be decreasing with time. This is reasonable since $\operatorname{Tr}\left(F_{j}\right)$ need not equal $\operatorname{Tr}\left(F_{n}\right)$.

B. Symmetries Possessed by Motions

A motion is said to possess the unitary, symnetry $U_{0}$ if, for all. $\rho \in S_{d}$,

$$
\mathscr{R}\left(t^{\prime \prime}, t^{\prime}\right) \cdot\left[U_{0} \rho\left(t^{\prime}\right) U_{0}^{+}\right]=U_{0} \rho\left(t^{\prime \prime}\right) U_{0}^{+}
$$

end is said to possess the antiunitary symmetry. $U_{0}$ if

$$
R\left(t^{\prime \prime}, t^{\prime}\right)\left[U_{0} \tilde{\rho}\left(t^{\prime}\right) U_{0}^{+}\right]=U_{0} \tilde{\rho}\left(t^{\prime \prime}\right) U_{0}^{+}
$$

That the motion has such a symmetry inplies, and is implied by:

(i)

Unitary symmetry

$Q_{0} R Q_{0}^{-1}=R$

Antiunitary symmetry

$$
Q_{0} J R S Q_{0}^{-1}=R
$$

where $Q_{0}$ and $Q_{0} \mathcal{T}$ are defined in section IV-A $(a)$.

Unitary symmetry

$$
\begin{aligned}
& Q_{0} m Q_{0}^{-1}=m \\
& Q_{0} \alpha Q_{0}^{-1}=\mathscr{L}
\end{aligned}
$$


Antiunitary symmetry

$$
\begin{aligned}
& Q_{0} \mathcal{T} \mathscr{T} Q_{0}^{-1}=m \\
& Q_{0} \mathscr{T} \mathscr{L} \mathcal{T} Q_{0}^{-1}=\mathscr{L}
\end{aligned}
$$

(c) For motions of type (C-2), for unitary symmetry, $P\left(u ; t^{\prime \prime}, t^{\prime}\right)$ may bo so. selected that

$$
P\left(u_{0} u u_{0}^{+} ; t^{\prime \prime}, t^{\prime}\right)=P\left(u ; t^{\prime \prime}, t^{\prime}\right)
$$

(It would be false to say that $P\left(u ; t^{\prime \prime}, t^{\prime}\right)$ is invariant, since these functions are not unique.) Likewise for motions of type (c-3),

$$
\mathrm{U}(\mathrm{g}) \rightarrow \mathrm{U}_{0} \mathrm{U}(\mathrm{g}) \mathrm{U}_{\mathrm{O}}^{+}
$$

is an automorphism, and $P\left(g ; t^{\prime \prime}, t^{\prime}\right)$ may bo so selected that it is invariant under this automorphism.

For an antiulitary symmetry, $P\left(u ; t^{\prime \prime}, t^{\prime}\right)$ may bo so selected that

$$
P\left(u_{0} u^{*} u_{0}^{+} ; t^{\prime \prime}, t^{\prime}\right)=E\left(u ; t^{\prime \prime}, t^{\prime}\right)
$$

inkewise, for motions of type (c.-3),

$$
\mathrm{U}(\mathrm{g}) \rightarrow \mathrm{U}_{\mathrm{O}} \mathrm{U}^{*}(\mathrm{~g}) \mathrm{U}_{\mathrm{O}}^{+}
$$

is also en automorphism, and $P\left(\varepsilon ; t^{\prime \prime}, t^{\prime}\right)$ may be so selected that it, is invariant under this automorphisin.

It is easy to see that if $U_{0}$ is a unitary (antiunitary) symetioy possessed by any rotion of type $(A)$, then the subspace of $V$ belonging to a given eigenvalue of $Q_{0}\left(Q_{0} \mathscr{T}\right)$ is stable under the notion. For 


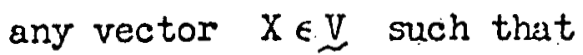

$$
\left.Q_{0} x=\lambda x \quad \text { (or, } Q_{0} \mathscr{J} x=\lambda x\right)
$$

one has, respectively,

$$
Q_{0}(R x)=\lambda(\mathbb{R} x) \quad\left(\text { or, } Q_{0} g(\mathbb{R})=\lambda(\mathbb{R} x)\right)
$$

Furthermore, $Q_{0} \rho$ (or $Q_{0} g \rho$ ) is stable if $\rho$ is. If a density. matrix is in the subspace belonging to a given eigenvalue of $Q_{0}$, the eigenvalue is necessarily unity, wich means that it commutes with $U_{0}$. One gets a reduced problem by considering the motion of all denșity matrices which commute with $U_{0}$. (Similarly, for an antiunitary symmetry, one may consider the motion of all density matrices satisfying $\left.U_{0} \tilde{\rho}_{0} U_{0}^{+}=\rho.\right)$ For exarple, in certain experinents involving optical pumping, one may have it so that the motion is invariant under rotations around the 3-2xis, in which case a diagonal density matrix in the representition where $\mathrm{J}_{3}$ is diagonal will stay diagonal, and one gets a reduced problem in studying the motion of diagonal density matrices. 
VIII. CONCLUDING REMTRS

We ruve studied stochastic motions from two viewpoints: we studied the" propertice of feineral linear mapings, and the corresponding motions, which .

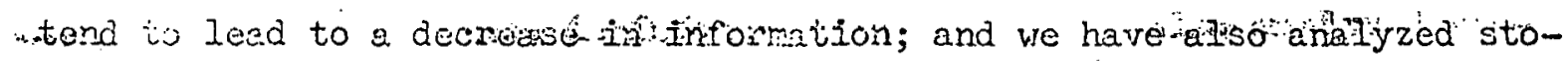
che to rotions hich correspond to a diffusion process on a group manifold. Sitholgh for simplicity and definiteness we have limited ourselves to the consideration of finite dimensional Hilbert spaces, it is clear that many results havo extensions to the infinite dinensional case. The difrusion description in particular provides a natural way of making such extensions. Tho derivation of $E_{q}$ (V-22) for $P(g ; t)$ has clearly nothing to do with tho Girnensionality of the Hilbert space of the system, and even in the derivation of Eq. (v-20) for the density matrix no use has been mide of the finite dinensionality of the Hilbert space on wich $\rho$ operates. The diffusion equation for the density matrix will therefore formaliy be exactly the samo for the infinite dinensional case. Of course in this case the integration of Eq. $(\mathrm{V}-2 \mathrm{O})$ is no longer as simple as in the finite dinensional case. Furthermore, it goes without seying that not everytring which holds for Iinite dimenional vector spaces will continue to hold. Thus, for instance, thetrace operation can be defined only with further specification on the .25 type of operators, and the definition of entropy becores obscure. Nevertheless, it is worth noting that the considaration of the motion of a dym mical variable may still lead to a finite system of ordinary differential equations, if a finito diniensional vector space spanned by dynamical variables

25. "Trece-type operator"s" Iom a proper subclass of lilbert-Schnidt operetors. For possible extensions of the definition of the trace operation ses I. E. Sogal, J. of lath. and Hech., 2, 623 (I960). 
is stable under the motion, as was mentioned under Eq. (VII-5). It is advantageous, therefore, to concentrate on the motion of dynamical variables in such cases. 
Appendix 1

\section{The Inversion Formula Eq.. (III-13)}

We will first give the following lemma:

Lemma: Let $X \in \sim_{h}$, and $\left\{B_{\alpha}\right\}$ be a complete set of orthonormal hermitean basis for $\mathrm{Y}$, then

$$
\sum_{\alpha=1}^{N^{2}} B_{\alpha} \times B_{\alpha}=I \operatorname{Tr}(x)
$$

This lemin is most easily proved by first using a special basis $e_{i j}$ and $o_{i j}$ of the form:

$$
\begin{aligned}
e_{i j} & =(2)^{-1 / 2}(|i>\langle j|+| j\rangle<i|) \text { for } i \neq j . \\
e_{i i} & =|i>i| \\
o_{i j} & =i(2)^{-1 / 2}(\mid i>\langle j|-| j>i|)
\end{aligned}
$$

The result is then generalized to other orthonormal bases by a real orthogonal transformation in $\mathcal{L Y}$.

By use of this lemna, the Eo. (III-13) and Eq. (III-15) are casily proved. 


\section{Appendix 2 .}

\section{Real Parts of the Eigenvalues of $m$}

For constant $M$, it is clear that the real parts of the eigenvalues of $7 n$ cannot be positive, since otherwise the exponential growth of the solution will take some density matrix out of the set of density matrices. We will show that the same restriction is necessary even when $m(t)$ is varying with time.

Let $\mathrm{y}(t)$ bo an eigenvector of $\mathbb{M}(t)$ corresponding to some eigenvalue $\lambda$. (It is to be noted that $\lambda$ may be a degenerate eigenvalue, and that $M(t)$ may not be diagonalizable, whereas $\mathcal{L}(t)$ can always be diagonalized.) Let $y(t)$ be normalized to unity, then

$$
\operatorname{Ro} \lambda=\sum_{\alpha, \beta=1}^{N^{2}} \operatorname{Re}\left(y_{\alpha} m_{\alpha \beta} y_{\beta}\right)
$$

or,

$$
\text { Re } \lambda=1 / 2 \sum_{\alpha, \beta}\left\{\mathcal{L}_{\alpha \beta} \operatorname{Tr}\left(B_{\alpha} Y_{\beta} Y^{+}\right)+\mathcal{L}_{\alpha \beta} \operatorname{Tr}\left(\mathrm{B}_{\alpha} \mathrm{Y}^{+} \mathrm{B}_{\beta} \mathrm{Y}\right)\right\}
$$

where $Y=\sum_{a=1}^{n^{2}} y_{\alpha} B_{\alpha}$, and is not necessarily diagonalizable.

Denote

$$
J=I / 2\left(Y+Y^{+}\right) ; \quad K=I / 2\left(Y-Y^{+}\right)
$$

We have

$$
\operatorname{Re} \lambda=\sum_{\alpha_{\nu} \beta} \mathcal{L}_{\alpha \beta}\left\{\operatorname{Tr}\left(B_{\alpha} J B_{\beta} J^{+}\right)+\operatorname{Tr}\left(B_{\alpha} K_{B} K^{+}\right)\right\}
$$

Both $\mathrm{J}$ and $\mathrm{K}$ can be expanded into prinitive hermitenn idompotents:

$$
J=\sum_{\mathrm{n}=1}^{N} j_{\mathrm{m} n} \mathrm{E}_{\mathrm{n}} ; \quad \because \mathrm{K}=\sum_{\mathrm{n}=1}^{N} i k_{\mathrm{n}}{ }_{\mathrm{n}}
$$

where $j_{m}$ and $k_{n}$ are real. 


$$
\begin{aligned}
& \text { Denoting the symmetric parts of } \sum_{\alpha, \beta} \mathscr{L}_{\alpha \beta} \operatorname{Tr}\left(B_{\alpha} E_{m} B_{\beta} E_{n}\right) \text { and } \\
& \sum_{\alpha, \beta} \mathcal{L}_{\alpha \beta} \operatorname{Tr}\left(B_{\alpha} E^{\prime} m_{\beta} E^{\prime}{ }_{n}\right) \text { by } f_{m n} \text { and }{ }_{f_{m n}^{\prime}} \text {, respectively, we have } \\
& \operatorname{Re} \lambda=\sum_{m, n=1}^{N}\left(j_{m} j_{n m}+k_{m} k_{n} f_{m n}^{\prime}\right) \\
& \text { Re } \lambda=\frac{1}{2} \sum_{\substack{m_{m}, n \\
\text { m } \neq n}}\left\{\left(j_{m}-j_{n}\right)\left(j_{n}-j_{m}\right) \hat{f}_{m n}+\left(k_{m}-k_{n}\right)\left(k_{n}-k_{m}\right) f_{m n}\right\} \leqslant 0 \quad(A 2-7)
\end{aligned}
$$

Hence for matrices $M$ in $M$ satisfying Eq. (III-18) and (III-19), the condition. (III-20) implies the regative semidefiniteness of the real parts of the eigenvalues of $\gamma \eta(t)$.

Fom Eq. (IV-10), we see that the condition (III-20) also implies that the eicenvalues of $R C \stackrel{L}{\sim}$ do not exceed one in magnitudo.

That the positure semidefinitoness of the submatrix $\mathcal{L}^{\circ}$ irplies condition (III-20) cen be shom in a similar mannor. Findly, that the arrows do not go to the left in Eq. (III-2I) can be demonstrated by counterexamples. 


\section{hpperdix 3}

Stable Ensembles

(a) Proof of the Eq. (III-26). We first give the following lemma.

Lemma: If $\Omega \in \mathscr{H}_{\mathrm{I}}$, and $F$ is a hermitean idempotient such that

$$
\operatorname{Tr}(F R F)=\operatorname{Tr}(F) \doteq f
$$

then, $\quad R F=F$.

This follows from,

$$
0 \leqslant \operatorname{Tr}(F-Q F)^{2}=\operatorname{Tr}(Q F, R F)-f \leqslant 0
$$

and hence

$$
\operatorname{Tr}(F-\mathfrak{R} F)^{2}=0
$$

No', for $X \in V_{h}$, which satisfies $Q P X=X$, let the expension of $X$ into hermitean idempotents be $x=\sum_{k=1}^{p} x_{k} F_{k}$, and such that $F_{k}$ are associated with distinct eigenvalues. We order the eigenvalues such that,

$$
x_{1}<x_{2}<\ldots<x_{p}
$$

Let us also define

$$
f_{k} \equiv \operatorname{Tr}\left(F_{k}\right)>0
$$

Then we have

$$
f_{p} x_{p}=\operatorname{Tr}\left(F_{p} X\right)=\sum_{k=1}^{p} x_{k} \operatorname{Tr}\left(F_{p} R F_{k}\right)
$$


or,

$$
f_{p} x_{p}=x_{p} \sum_{k=1}^{p} \operatorname{Tr}\left(F_{p} R F_{k}\right)-\sum_{k=1}^{p-I}\left(x_{p}-x_{k}\right) \operatorname{Tr}\left(F_{p} R F_{k}\right)
$$

i.e.

$$
f_{p} x_{p}=f_{p} x_{p}-\sum_{k=1}^{p-1}\left(x_{p}-x_{k}\right) \operatorname{Tr}\left(F_{p} Q F_{k}\right)
$$

Since each term in the sum is non-negative, we must have

$$
\operatorname{Tr}\left(F_{p} \cdot Q F_{k}\right)=0 \quad \text { for } k<p
$$

Hence

$$
\operatorname{Tr}\left(F_{p} R F_{p}\right)=f_{p}
$$

Also, sinco each term in the sum $\sum_{k=1}^{p} \operatorname{Tr}\left(F_{k} R F_{p}\right)$ is non-negative, we. have

$$
\operatorname{Tr}\left(F_{k} R F_{p}\right)=0 . \quad \text { for } k<p
$$

The argument can then be repeated for $f_{p-I^{2}}{ }_{p-I}$, and so forth. Eq. (III-26) then follows from the lemm $(43-2)$. The corollary follows from the definjtion of the matrices $M$.

(b) Proci of Eq. (III-28).

Let $Q \mathcal{R} \in \mathcal{Q}, x \in y_{h}$, and $R x=x$. Let the exounsion of $x$ into primitive hermitean idempotents be such that $x=\sum_{k=1}^{n} x_{k} E_{k}$, where $x \geqslant 0$ for $I \leqslant k \leqslant n$. ' and $x<0$ for $p<k \leqslant n$.

Denote

$$
P \equiv \sum_{k=1}^{p} x_{k} E_{k} ; \quad N \equiv \sum_{k=p+1}^{n} x_{k} E_{k}
$$

If

$$
R P=P+D
$$


then

$$
a N=N-D
$$

We havo

$$
\operatorname{Tr}\left(E_{k} \cap P\right)+\operatorname{Tr}\left(E_{k} \cap N\right)=x_{l}
$$

Since

$$
\operatorname{Tr}\left(\sum_{k} \cap \mathbb{N}\right) \leq 0
$$

it means that

$$
\operatorname{Tr}\left(F_{k} R P\right) \geqslant x_{k}
$$

On the other hend,

$$
\sum_{k=1}^{p} \operatorname{Tr}\left(\sum_{k} Q P\right)+\sum_{k=0+1}^{n} \operatorname{mr}\left(\sum_{k} Q P\right)=\sum_{k=1}^{n} x_{k}
$$

and

$$
\sum_{k=p+1}^{n} \operatorname{Tr}\left(E_{k} R \dot{P}\right) \geqslant 0
$$

Hence the equality sign holds in Eq. (k3-10) ior $I \leqslant k \leqslant 0$. This mons that

$$
E_{k} R N=0 \quad(i: \leqslant p)
$$

which in turn means

$$
E_{k} D=0 \quad(! \leqslant 0)
$$

Similarly, it can be seen that $E_{k} D=0$ for $p<k \leqslant n$ also. Hence,

$$
D \equiv 0
$$




\section{Acknowledgements}

It is a great pleasure to express the author's gratitude to Professor E. H. Wichmann, who suggested tho present study, and provided inspiration and guidince throughout the coursg of his work. Ho is grateful to Profossor M. A. Ruderman for advices and encouragement. He also wishes to thanle Professor B. Fledman, Hrotessor I. Fdry, Frofessur J. Feldman, Dr. R. R. Phelps, and $J$. de Pillis of the Mathematics Departriont at the University of California for helpful discussions and advice. Finally, Iinancial assistance from the U. S. Atomic Energy Commission and the Mational Science Foundation is gratefully acknowledged. 
This report was prepared as an account of Government sponsored work. Neither the United States, nor the Commission, nor any person acting on behalf of the Commission:

A. Makes any warranty or representation, expressed or implied, with respect to the accuracy, completeness, or usefulness of the information contained in this report, or that the use of any information, apparatus, method, or process disclosed in this report may not infringe privately owned rights; or

B. Assumes any liabilities with respect to the use of, or for damages resulting from the use of any information, apparatus, method, or process disclosed in this report.

As used in the above, "person acting on behalf of the Commission" includes any employee or contractor of the Commission, or employee of such contractor, to the extent that such employee or contractor of the Commission, or employee of such contractor prepares, disseminates, or provides access to, any information pursuant to his employment or contract with the Commission, or his employment with such contractor. 